\title{
A Novel Method for Multiattribute Decision Making with Dual Hesitant Fuzzy Triangular Linguistic Information
}

\author{
Yanbing Ju, Shanghong Yang, and Xiaoyue Liu \\ School of Management and Economics, Beijing Institute of Technology, Beijing 100081, China \\ Correspondence should be addressed to Yanbing Ju; juyb@bit.edu.cn
}

Received 5 November 2013; Accepted 11 January 2014; Published 27 February 2014

Academic Editor: Kazutake Komori

Copyright (C) 2014 Yanbing Ju et al. This is an open access article distributed under the Creative Commons Attribution License, which permits unrestricted use, distribution, and reproduction in any medium, provided the original work is properly cited.

\begin{abstract}
This paper studies the multiattribute decision making (MADM) problems in which the attribute values take the form of dual hesitant fuzzy triangular linguistic elements and the weights of attributes take the form of real numbers. Firstly, to solve the situation where the membership degree and the nonmembership degree of an element to a triangular linguistic variable, the concept, operational laws, score function, and accuracy function of dual hesitant fuzzy triangular linguistic elements (DHFTLEs) are defined. Then, some dual hesitant fuzzy triangular linguistic geometric aggregation operators are developed for aggregating the DHFTLEs, including dual hesitant fuzzy triangular linguistic weighted geometric (DHFTLWG) operator, dual hesitant fuzzy triangular linguistic ordered weighted geometric (DHFTLOWG) operator, dual hesitant fuzzy triangular linguistic hybrid geometric (DHFTLHG) operator, generalized dual hesitant fuzzy triangular linguistic weighted geometric (GDHFTLWG) operator, and generalized dual hesitant fuzzy triangular linguistic ordered weighted geometric (GDHFTLOWG) operator. Furthermore, some desirable properties of these operators are investigated in detail. Based on the proposed operators, an approach to MADM with dual hesitant fuzzy triangular linguistic information is proposed. Finally, a numerical example for investment alternative selection is given to illustrate the application of the proposed method.
\end{abstract}

\section{Introduction}

Multiattribute decision making (MADM) has become a hot research topic, which is to select the most desirable solution from a finite set of feasible alternatives with respect to conflicting attributes, both quantitative and qualitative. Due to the increasing complexity of the socioeconomic environment and the lack of knowledge or data about the decision making problems domain, the attributes involved in the decision problems are not always expressed as crisp numbers, and many researchers have utilized the fuzzy theory to deal with investment alternative selection [1-4]. The fuzzy set theory originally proposed by Zadeh [5] is a very useful tool to describe uncertain information. However, in some real decision situations the fuzzy set is imprecise resulting from characterizing the fuzziness just by a membership degree. On the basis of the fuzzy set theory, Atanassov $[6,7]$ proposed the intuitionistic fuzzy set characterized by a membership function and a nonmembership function. Obviously, the intuitionistic fuzzy set can describe and characterterize the fuzzy essence of the objective world more exquisitely, and it has received more and more attention since its appearance [8-13].

However, in the real world, decision makers usually cannot completely express their opinions by quantitative numbers, and some of them are more appropriately described by qualitative linguistic terms. Since linguistic variables [14] have been proposed, so far, a number of linguistic approaches have been defined such as 2-tuple linguistic [15], interval-valued 2-tuple linguistic [16], uncertain linguistic [17], and trapezoid fuzzy linguistic [18]. In order to express the uncertainty and ambiguity as accurate as possible, Wang and $\mathrm{Li}$ [19] proposed the concept of intuitionistic linguistic set based on linguistic variables and intuitionistic fuzzy set, which can overcome the defects for intuitionistic fuzzy set which can only roughly represent criteria's membership and nonmembership to a particular concept, such as "good" and "bad," and for linguistic variables which usually implies that membership degree is 1 , and the nonmembership degree and hesitation degree of decision makers cannot be expressed. 
Furthermore, Liu and Jin [20] and Liu [21] proposed the intuitionistic uncertain linguistic variables and the intervalvalued intuitionistic uncertain linguistic variables as well as some decision making methods.

In real decision making process, we often encounter such situation that the decision makers are hesitant among a set of possible values which makes the outcome of decision making inconsistent. To solve this problem, the hesitant fuzzy set (HFS), an extension of fuzzy set [5], was proposed by Torra and Narukawa [22] and Torra [23], which permits the membership degree of an element to a given set to be represented by several possible numerical values. To accommodate more complex environment, several extensions of HFS have been presented, such as interval-valued hesitant fuzzy set (IVHFS) $[24,25]$ and hesitant fuzzy linguistic term set (HFLS) [26]. Especially, considering that the human judgments including preference information may be stated which permits the membership having a set of possible hesitant fuzzy linguistic values or hesitant fuzzy uncertain linguistic values, Lin et al. [27] proposed the concepts of hesitant fuzzy linguistic set (HFLS) and hesitant fuzzy uncertain linguistic set (HFULS). Furthermore, some aggregation operators hesitant fuzzy linguistic weighted average (HFLWA) operator, hesitant fuzzy linguistic ordered weighted average (HFLOWA) operator, hesitant fuzzy uncertain linguistic weighted average (HFULWA) operator, hesitant fuzzy uncertain linguistic ordered weighted average (HFULOWA) operator, and an approach are proposed for MADM problems. However, the above hesitant fuzzy decision making methods just provide the membership degrees and neglect the importance of the nonmembership degrees. In fact, the nonmembership plays the same important role as the membership in describing the vague decision making information, which indicates that the possible degrees of one element do not belong to a fixed set. To assess the attribute values more precisely, Zhu et al. [28] developed the dual hesitant fuzzy set (DHFS), taking much more information into account given by decision makers, in which the membership degree and the nonmembership degree are in the form of sets of values in $[0,1]$. Then correlation coefficient $[29,30]$ and aggregation operators [31] are proposed to deal with MADM problems under dual hesitant fuzzy environment. Furthermore, Ju et al. [32] proposed the definition of the interval-valued dual hesitant fuzzy set (IVDHFS).

To the best of our knowledge, the existing approaches under the hesitant fuzzy environment are not suitable for dealing with MADM problems with dual hesitant fuzzy triangular linguistic information. Therefore, motivated by the idea of HFLS and HFULS, based on the triangular linguistic term set and the DHFS, in this paper, we define a new concept called the dual hesitant fuzzy triangular linguistic set composed of a triangular linguistic term, a set of membership degrees, and a set of nonmembership degrees, which can overcome the shortcomings of the HFLS. For example, for a predefined linguistic set $S=\left\{s_{0}=\right.$ extremely low, $s_{1}=$ very low, $s_{2}=$ low, $s_{3}=$ medium, $s_{4}=$ high, $s_{5}=$ very high, $s_{6}=$ extremely high\}, we can evaluate the "growth" of a company by a dual hesitant fuzzy triangular linguistic element (DHFTLE) $\left\langle\left[s_{3}, s_{4}, s_{5}\right],\{0.4,0.5,0.6\},\{0.2,0.3,0.4\}\right\rangle$.
This is the motivation of our study. The main advantages of DHFTLE include (1) triangular linguistic term which can describe the uncertainty more precisely and objectively than linguistic term and uncertain linguistic term in qualitative; (2) a set of membership degrees and a set of nonmembership degrees are complements of the triangular linguistic terms, which can explain how much degree that an attribute value belongs to and not belong to a triangular linguistic term in quantitative.

The remainder of this paper is organized as follows. Some basic definitions of triangular linguistic term set, hesitant fuzzy set, and dual hesitant fuzzy set are briefly reviewed in Section 2. In Section 3, the concept, operational laws, score function, and accuracy function of the dual hesitant fuzzy triangular linguistic elements are defined. In Section 4, some dual hesitant fuzzy triangular linguistic geometric aggregation operators are proposed, and then some desirable properties of the proposed operators are investigated. In Section 5, we develop an approach for multiple attribute decision making with dual hesitant fuzzy triangular linguistic information based on the proposed operators. In Section 6, a numerical example is given to illustrate the application of the proposed method. The paper is concluded in Section 7.

\section{Preliminaries}

To facilitate the following discussion, some basic definitions related to triangular linguistic term set, hesitant fuzzy set, and dual hesitant fuzzy set are briefly reviewed in this section.

2.1. Triangular Linguistic Term Set. Let $S=\left\{s_{0}, s_{1}, \ldots, s_{l}\right\}$ be a finite linguistic term set with odd cardinality, where $s_{i}$ represents a possible value for a linguistic term and $l+1$ is the cardinality of $S$. For example, when $l=6$, a set of seven terms $S$ could be given as follows:

$$
\begin{aligned}
& S=\left\{s_{0}=\text { extremely low, } s_{1}=\text { very low, } s_{2}=\text { low, } s_{3}\right. \\
& =\text { medium, } s_{4}=\text { high, } s_{5}=\text { very high, } s_{6}=\text { extremely } \\
& \text { high }\} .
\end{aligned}
$$

In general, for any linguistic term set $S$, it is required that $s_{i}$ and $s_{j}$ satisfy the following properties $[33,34]$ :

(1) the set is ordered: $s_{i}>s_{j}$, if and only if $i>j$;

(2) there is the negation operator: $\operatorname{Neg}\left(s_{i}\right)=s_{j}$, such that $j=g-i$;

(3) maximum operator: $\max \left\{s_{i}, s_{j}\right\}=s_{i}$, if $i \geq j$;

(4) minimum operator: $\min \left\{s_{i}, s_{j}\right\}=s_{j}$, if $i \geq j$.

Definition 1 (see [35]). Let $\bar{S}=\left\{s_{i} \mid s_{0} \leq s_{i} \leq s_{g}, i \in[0, g]\right\}$ be the continuous form of $S, s_{\theta}, s_{\pi}, s_{\tau} \in \bar{S}$, and $s_{\theta} \leq s_{\pi} \leq s_{\tau}$; then $\widetilde{s}=\left[s_{\theta}, s_{\pi}, s_{\tau}\right]$ can be called a triangular linguistic variable, and $\widetilde{S}$ is called a triangular linguistic term set.

Especially, if $s_{\theta}=s_{\pi}=s_{\tau}$, then the triangular linguistic variable $\widetilde{s}$ reduces to a linguistic variable; if $s_{\theta}=s_{\pi}$ or $s_{\pi}=s_{\tau}$, then the triangular linguistic variable $\widetilde{s}$ reduces to an uncertain linguistic variable. 
Let $\widetilde{S}$ be a set of triangular linguistic variables; for any two triangular linguistic variables $\widetilde{s}_{1}=\left[s_{\theta_{1}}, s_{\pi_{1}}, s_{\tau_{1}}\right]$ and $\widetilde{s}_{2}=$ $\left[s_{\theta_{2}}, s_{\pi_{2}}, s_{\tau_{2}}\right], \widetilde{s}_{1}, \widetilde{s}_{2} \in \widetilde{S}$, the operational laws are shown as follows [35]:

$$
\begin{aligned}
& \text { (1) } \widetilde{s}_{1} \oplus \widetilde{s}_{2}=\left[s_{\theta_{1}}, s_{\pi_{1}}, s_{\tau_{1}}\right] \oplus\left[s_{\theta_{2}}, s_{\pi_{2}}, s_{\tau_{2}}\right]=\left[s_{\theta_{1}+\theta_{2}}, s_{\pi_{1}+\pi_{2}},\right. \\
& \left.s_{\tau_{\tau_{1}+\tau_{2}}}\right] ; \\
& \text { (2) } \widetilde{s}_{1} \otimes \widetilde{s}_{2}=\left[s_{\theta_{1}}, s_{\pi_{1}}, s_{\tau_{1}}\right] \otimes\left[s_{\theta_{2}}, s_{\pi_{2}}, s_{\tau_{2}}\right]=\left[s_{\theta_{1} \times \theta_{2}}, s_{\pi_{1} \times \pi_{2}},\right. \\
& \left.s_{\tau_{1} \times \tau_{2}}\right] ; \\
& \text { (3) } \widetilde{s}_{1}^{\beta}=\left[s_{\theta_{1}}, s_{\pi_{1}}, s_{\tau_{1}}\right]^{\beta}=\left[s_{\left(\theta_{1}\right)^{\beta}}, s_{\left(\pi_{1}\right)^{\beta}}, s_{\left(\tau_{1}\right)}\right], \beta>0 ; \\
& \text { (4) } \beta \widetilde{s}_{1}=\beta\left[s_{\theta_{1}}, s_{\pi_{1}}, s_{\tau_{1}}\right]=\left[s_{\beta \theta_{1}}, s_{\beta \pi_{1}}, s_{\beta \tau_{1}}\right], \beta>0 .
\end{aligned}
$$

\subsection{Hesitant Fuzzy Set}

Definition 2 (see $[22,23])$. Let $X$ be a fixed set; then a hesitant fuzzy set (HFS) on $X$ is in terms of a function $h$ that when applied to $X$ returns a subset of $[0,1]$, which can be represented as the following mathematical symbol:

$$
E=\{\langle x, h(x)\rangle \mid x \in X\},
$$

where $h(x)$ is a set of some values in $[0,1]$, denoting the possible degrees of the element $x \in X$ to the set $E$. For convenience, Xia and Xu [36] called $h(x)$ a hesitant fuzzy element (HFE) and $E$ the set of all hesitant fuzzy elements (HFEs).

Definition 3 (see [36]). Let $h$ be a HFE; then the score function of $h$ is determined as follows:

$$
S(h)=\frac{1}{\# h} \sum_{r \in h} r,
$$

where $\# h$ is the number of the elements in $h$. For two HFEs $h_{1}$ and $h_{2}$, if $S\left(h_{1}\right)>S\left(h_{2}\right)$, then $h_{1}>h_{2}$; if $S\left(h_{1}\right)=S\left(h_{2}\right)$, then $h_{1}=h_{2}$.

\subsection{Dual Hesitant Fuzzy Set}

Definition 4 (see [28]). Let $X$ be a fixed set; then a dual hesitant fuzzy set (DHFS) $\widetilde{D}$ on $X$ is described as

$$
\widetilde{D}=\{\langle x, \widetilde{h}(x), \widetilde{g}(x)\rangle \mid x \in X\},
$$

in which $\widetilde{h}(x)$ and $\widetilde{g}(x)$ are two sets of some values in $[0,1]$, denoting the possible membership degrees and nonmembership degrees of the element $x \in X$ to the set $\widetilde{D}$, respectively, with the conditions

$$
0 \leq r, \quad \eta \leq 1, \quad 0 \leq r^{+}+\eta^{+} \leq 1,
$$

where $r \in \widetilde{h}(x), \eta \in \widetilde{g}(x), r^{+} \in \widetilde{h}^{+}(x)=\cup_{r \in \widetilde{h}(x)} \max \{r\}$, and $\eta^{+} \in \widetilde{g}^{+}(x)=\cup_{\eta \in \widetilde{g}(x)} \max \{\eta\}$ for all $x \in X$. For convenience, the pair $\widetilde{d}(x)=\langle\widetilde{h}(x), \widetilde{g}(x)\rangle$ is called a dual hesitant fuzzy element (DHFE) denoted by $\widetilde{d}=\langle\widetilde{h}, \widetilde{g}\rangle$.

Obviously, if there is only one element in both $\widetilde{h}(x)$ and $\tilde{g}(x)$, the DHFE reduces to an intuitionistic fuzzy number [6].
Definition 5 (see [28]). Let $\widetilde{d}_{i}=\left\langle\widetilde{h}\left(\widetilde{d}_{i}\right), \widetilde{g}\left(\widetilde{d}_{i}\right)\right\rangle(i=1,2, \ldots, n)$ be a collection of DHFEs, then the score function $S\left(\widetilde{d}_{i}\right)$ and the accuracy function $P\left(\widetilde{d}_{i}\right)$ of $\widetilde{d}_{i}(i=1,2, \ldots, n)$ can be defined by (5) and (6), respectively;

$$
\begin{aligned}
& S\left(\widetilde{d}_{i}\right)=\frac{1}{\# \widetilde{h}} \sum_{r \in \widetilde{h}} r-\frac{1}{\# \widetilde{g}} \sum_{\eta \in \widetilde{g}} \eta, \\
& P\left(\widetilde{d}_{i}\right)=\frac{1}{\# \widetilde{h}} \sum_{r \in \tilde{h}} r+\frac{1}{\# \widetilde{g}} \sum_{\eta \in \widetilde{g}} \eta,
\end{aligned}
$$

where $\# \widetilde{h}$ and $\# \widetilde{g}$ are the numbers of values in $\widetilde{h}\left(\widetilde{d_{i}}\right)$ and $\widetilde{g}\left(\widetilde{d_{i}}\right)$, respectively.

\section{Dual Hesitant FuzzyTriangular Linguistic Set}

Based on the triangular linguistic term set and the dual hesitant fuzzy set, we propose the definition of the dual hesitant fuzzy triangular linguistic set, the operational laws, score function, and accuracy function in what follows.

Definition 6. Let $X$ be a fixed set; then a dual hesitant fuzzy triangular linguistic set (DHFTLS) $D$ on $X$ is described as

$$
D=\left\{\left\langle x\left[s_{\theta(x)}, s_{\pi(x)}, s_{\tau(x)}\right], h(x), g(x)\right\rangle \mid x \in X\right\},
$$

in which $\left[s_{\theta(x)}, s_{\pi(x)}, s_{\tau(x)}\right] \in \widetilde{S}, h(x)$ and $g(x)$ are two sets of some values in $[0,1]$, denoting the possible membership degrees and nonmembership degrees of the element $x \in X$ to the triangular linguistic variable $\left[s_{\theta(x)}, s_{\pi(x)}, s_{\tau(x)}\right]$, respectively, with the conditions

$$
0 \leq r, \quad \eta \leq 1, \quad 0 \leq r^{+}+\eta^{+} \leq 1,
$$

where $r \in h(x), \eta \in g(x), r^{+} \in h^{+}(x)=\cup_{r \in h(x)} \max \{r\}$, and $\eta^{+} \in g^{+}(x)=\cup_{\eta \in g(x)} \max \{\eta\}$ for all $x \in X$. For convenience, the 3-tuples $d(x)=\left\langle\left[s_{\theta(x)}, s_{\pi(x)}, s_{\tau(x)}\right], h(x), g(x)\right\rangle$ is called a dual hesitant fuzzy triangular linguistic element (DHFTLE) denoted by $d=\left\langle\left[s_{\theta}, s_{\pi}, s_{\tau}\right], h, g\right\rangle$.

Especially, if $s_{\theta}=s_{\pi}=s_{\tau}$, then the dual hesitant fuzzy triangular linguistic element $d=\left\langle\left[s_{\theta}, s_{\pi}, s_{\tau}\right], h, g\right\rangle$ reduces to a dual hesitant fuzzy linguistic element; if $s_{\theta}=s_{\pi}$ or $s_{\pi}=s_{\tau}$, then the dual hesitant fuzzy triangular linguistic element $d=$ $\left\langle\left[s_{\theta}, s_{\pi}, s_{\tau}\right], h, g\right\rangle$ reduces to a dual hesitant fuzzy uncertain linguistic element.

Definition 7. Let $d_{1}=\left\langle\left[s_{\theta\left(d_{1}\right)}, s_{\pi\left(d_{1}\right)}, s_{\tau\left(d_{1}\right)}\right], h_{1}, g_{1}\right\rangle$ and $d_{2}=\left\langle\left[s_{\theta\left(d_{2}\right)}, s_{\pi\left(d_{2}\right)}, s_{\tau\left(d_{2}\right)}\right], h_{2}, g_{2}\right\rangle$ be two DHFTLEs; then the operational laws are defined as

(1) $d_{1} \oplus d_{2}=\left\langle\left[s_{\theta\left(d_{1}\right)+\theta\left(d_{2}\right)}, s_{\pi\left(d_{1}\right)+\pi\left(d_{2}\right)}, s_{\tau\left(d_{1}\right)+\tau\left(d_{2}\right)}\right]\right.$, $\left.\bigcup_{r_{1} \in h_{1}, r_{2} \in h_{2}, \eta_{1} \in g_{1}, \eta_{2} \in g_{2}}\left\{\left\{r_{1}+r_{2}-r_{1} r_{2}\right\},\left\{\eta_{1} \eta_{2}\right\}\right\}\right\rangle ;$

(2) $d_{1} \otimes d_{2}=\left\langle\left[s_{\theta\left(d_{1}\right) \times \theta\left(d_{2}\right)}, s_{\pi\left(d_{1}\right) \times \pi\left(d_{2}\right)}, s_{\tau\left(d_{1}\right) \times \tau\left(d_{2}\right)}\right]\right.$, $\left.\bigcup_{r_{1} \in h_{1}, r_{2} \in h_{2}, \eta_{1} \in g_{1}, \eta_{2} \in g_{2}}\left\{\left\{r_{1} r_{2}\right\},\left\{\eta_{1}+\eta_{2}-\eta_{1} \eta_{2}\right\}\right\}\right\rangle ;$ 
(3) $\lambda d_{1}=\left\langle\left[s_{\lambda \theta\left(d_{1}\right)}, s_{\lambda \pi\left(d_{1}\right)}, s_{\lambda \tau\left(d_{1}\right)}\right], \bigcup_{r_{1} \in h_{1}, \eta_{1} \in g_{1}}\{\{1-(1-\right.$ $\left.\left.\left.\left.r_{1}\right)^{\lambda}\right\},\left\{\left(\eta_{1}\right)^{\lambda}\right\}\right\}\right\rangle, \lambda>0$

(4) $d_{1}^{\lambda}=\left\langle\left[s_{\theta\left(d_{1}\right)^{\lambda}}, s_{\pi\left(d_{1}\right)^{\lambda}}, s_{\tau\left(d_{1}\right)^{\lambda}}\right], \bigcup_{r_{1} \in h_{1}, \eta_{1} \in g_{1}}\left\{\left\{\left(r_{1}\right)^{\lambda}\right\},\{1-\right.\right.$ $\left.\left.\left.\left(1-\eta_{1}\right)^{\lambda}\right\}\right\}\right\rangle, \lambda>0$.

Theorem 8. Let $d_{1}=\left\langle\left[s_{\theta\left(d_{1}\right)}, s_{\pi\left(d_{1}\right)}, s_{\tau\left(d_{1}\right)}\right], h_{1}, g_{1}\right\rangle$ and $d_{2}=$ $\left\langle\left[s_{\theta\left(d_{2}\right)}, s_{\pi\left(d_{2}\right)}, s_{\tau\left(d_{2}\right)}\right], h_{2}, g_{2}\right\rangle$ be two DHFTLEs; the operational laws of DHFTLEs are defined as follows:

(1) $d_{1} \oplus d_{2}=d_{2} \oplus d_{1}$;

(2) $d_{1} \otimes d_{2}=d_{2} \otimes d_{1}$;

(3) $\lambda d_{1} \oplus \lambda d_{2}=\lambda\left(d_{1} \oplus d_{2}\right), \lambda>0$;

(4) $d_{1}^{\lambda} \otimes d_{2}^{\lambda}=\left(d_{1} \otimes d_{2}\right)^{\lambda}, \lambda>0$.

Definition 9. Let $d=\left\langle\left[s_{\theta}, s_{\pi}, s_{\tau}\right], h, g\right\rangle$ be a DHFTLE; then the score function of $d$ is defined as follows:

$$
S(d)=\frac{\theta+2 \pi+\tau}{4 l} \times\left(\frac{1}{\# h} \sum_{r \in h} r-\frac{1}{\# g} \sum_{\eta \in g} \eta\right),
$$

where $\# h$ and $\# g$ are the numbers of values in $h$ and $g$, respectively. $(l+1)$ is the cardinality of $S=\left\{s_{0}, s_{1}, \ldots, s_{l}\right\}$.

Definition 10. Let $d=\left\langle\left[s_{\theta}, s_{\pi}, s_{\tau}\right], h, g\right\rangle$ be a DHFTLE; then the accuracy function of $d$ is defined as follows:

$$
P(d)=\frac{\theta+2 \pi+\tau}{4 l} \times\left(\frac{1}{\# h} \sum_{r \in h} r+\frac{1}{\# g} \sum_{\eta \in g} \eta\right),
$$

where $\# h$ and $\# g$ are the numbers of values in $h$ and $g$, respectively. $(l+1)$ is the cardinality of $S=\left\{s_{0}, s_{1}, \ldots, s_{l}\right\}$.

Theorem 11. Let $d_{1}=\left\langle\left[s_{\theta\left(d_{1}\right)}, s_{\pi\left(d_{1}\right)}, s_{\tau\left(d_{1}\right)}\right], h_{1}, g_{1}\right\rangle$ and $d_{2}=$ $\left\langle\left[s_{\theta\left(d_{2}\right)}, s_{\pi\left(d_{2}\right)}, s_{\tau\left(d_{2}\right)}\right], h_{2}, g_{2}\right\rangle$ be two DHFTLEs; they can be compared by the following rules:

(1) if $S\left(d_{1}\right)>S\left(d_{2}\right)$, then $d_{1}>d_{2}$;

(2) if $S\left(d_{1}\right)=S\left(d_{2}\right)$, then

$$
\begin{aligned}
& \text { if } P\left(d_{1}\right)>P\left(d_{2}\right) \text {, then } d_{1}>d_{2} \text {; } \\
& \text { if } P\left(d_{1}\right)=P\left(d_{2}\right) \text {, then } d_{1}=d_{2} .
\end{aligned}
$$

\section{Some Dual Hesitant Fuzzy Triangular Linguistic Geometric Aggregation Operators}

In what follows, based on the operational laws of DHFTLEs, we will develop some geometric aggregation operators for aggregating the dual hesitant fuzzy triangular linguistic information.

Definition 12. Let $d_{j}=\left\langle\left[s_{\theta\left(d_{j}\right)}, s_{\pi\left(d_{j}\right)}, s_{\tau\left(d_{j}\right)}\right], h_{j}, g_{j}\right\rangle(j=$ $1,2, \ldots, n)$ be a collection of DHFTLEs, and DHFTLWG : $\Omega^{n} \rightarrow \Omega$; then the dual hesitant fuzzy triangular linguistic weighted geometric (DHFTLWG) operator can be defined as

$$
\operatorname{DHFTLWG}\left(d_{1}, d_{2}, \ldots, d_{n}\right)=\prod_{j=1}^{n}\left(d_{j}\right)^{w_{j}},
$$

in which $\Omega$ is a dual hesitant fuzzy triangular linguistic set and $w=\left(w_{1}, w_{2}, \ldots, w_{n}\right)^{T}$ is the weight vector of $d_{j}(j=$ $1,2, \ldots, n)$, such that $w_{j} \in[0,1]$ and $\sum_{j=1}^{n} w_{j}=1$.

Especially, if $w=(1 / n, 1 / n, \ldots, 1 / n)^{T}$, then the DHFTLWG operator reduces to the dual hesitant fuzzy triangular linguistic geometric (DHFTLG) operator.

Theorem 13. Let $d_{j}=\left\langle\left[s_{\theta\left(d_{j}\right)}, s_{\pi\left(d_{j}\right)}, s_{\tau\left(d_{j}\right)}\right], h_{j}, g_{j}\right\rangle(j=$ $1,2, \ldots, n)$ be a collection of DHFTLEs and let $w=\left(w_{1}, w_{2}\right.$, $\left.\ldots, w_{n}\right)^{T}$ be the weight vector of $d_{j}(j=1,2, \ldots, n)$, such that $w_{j} \in[0,1]$ and $\sum_{j=1}^{n} w_{j}=1$. Then their aggregated value by the DHFTLWG operator is still a DHFTLE, and

$$
\begin{aligned}
& \operatorname{DHFTLWG}\left(d_{1}, d_{2}, \ldots, d_{n}\right) \\
& =\left\langle\left[ s_{\prod_{j=1}^{n}\left(\theta\left(d_{j}\right)\right)^{w_{j}}}, s_{\left.\prod_{j=1}^{n}\left(\pi\left(d_{j}\right)\right)^{w_{j}}, s_{\prod_{j=1}^{n}\left(\tau\left(d_{j}\right)\right)^{w_{j}}}\right],}\right.\right. \\
& \left.\bigcup_{r_{j} \in h_{j}, \eta_{j} \in \mathcal{g}_{j}}\left\{\left\{\prod_{j=1}^{n}\left(r_{j}\right)^{w_{j}}\right\},\left\{1-\prod_{j=1}^{n}\left(1-\eta_{j}\right)^{w_{j}}\right\}\right\}\right\rangle .
\end{aligned}
$$

Theorem 14 (boundedness). Let $d_{j}=$ $\left\langle\left[s_{\theta\left(d_{j}\right)}, s_{\pi\left(d_{j}\right)}, s_{\tau\left(d_{j}\right)}\right], h_{j}, g_{j}\right\rangle(j=1,2, \ldots, n)$ be a collection of DHFTLEs, for the DHFTLWG operator, if $s_{\theta^{-}}=\min _{1 \leq j \leq n} s_{\theta\left(d_{j}\right)}$, $s_{\pi^{-}}=\min _{1 \leq j \leq n} s_{\pi\left(d_{j}\right)}, s_{\tau^{-}}=\min _{1 \leq j \leq n} s_{\tau\left(d_{j}\right)}, s_{\theta^{+}}=$ $\max _{1 \leq j \leq n} s_{\theta\left(d_{j}\right)}, s_{\pi^{+}}=\max _{1 \leq j \leq n} s_{\pi\left(d_{j}\right)}, s_{\tau^{+}}=\max _{1 \leq j \leq n} s_{\tau\left(d_{j}\right)}$, $r^{-}=\min _{1 \leq j \leq n}\left\{r_{j} \mid r_{j} \in h_{j}\right\}, r^{+}=\max _{1 \leq j \leq n}\left\{r_{j} \mid r_{j} \in h_{j}\right\}$, $\eta^{-}=\min _{1 \leq j \leq n}\left\{\eta_{j} \mid \eta_{j} \in g_{j}\right\}, \eta^{+}=\max _{1 \leq j \leq n}\left\{\eta_{j} \mid \eta_{j} \in g_{j}\right\}$, for all $j=1,2, \ldots, n$; then one can obtain

$$
\begin{aligned}
\left\langle\left[s_{\theta^{-}}, s_{\pi^{-}}, s_{\tau^{-}}\right],\left\{r^{-}\right\},\left\{\eta^{+}\right\}\right\rangle & \leq \operatorname{DHFTLWG}\left(d_{1}, d_{2}, \ldots, d_{n}\right) \\
& \leq\left\langle\left[s_{\theta^{+}}, s_{\pi^{+}}, s_{\tau^{+}}\right],\left\{r^{+}\right\},\left\{\eta^{-}\right\}\right\rangle .
\end{aligned}
$$

Proof. Since $r^{-}=\min _{1 \leq j \leq n}\left\{r_{j} \mid r_{j} \in h_{j}\right\}, r^{+}=\max _{1 \leq j \leq n}\left\{r_{j} \mid\right.$ $\left.r_{j} \in h_{j}\right\}, \eta^{-}=\min _{1 \leq j \leq n}\left\{\eta_{j} \mid \eta_{j} \in g_{j}\right\}, \eta^{+}=\max _{1 \leq j \leq n}\left\{\eta_{j} \mid \eta_{j} \epsilon\right.$ $\left.g_{j}\right\}$, then for any $r_{j} \in h_{j}$ and $\eta_{j} \in g_{j}, j=1,2, \ldots, n$, we have

$$
r^{-} \leq r_{j} \leq r^{+}, \quad \eta^{-} \leq \eta_{j} \leq \eta^{+}, \quad j=1,2, \ldots, n ;
$$

then

$$
\begin{gathered}
\prod_{j=1}^{n}\left(r_{j}\right)^{w_{j}} \geq \prod_{j=1}^{n}\left(r^{-}\right)^{w_{j}}=r^{-}, \\
1-\prod_{j=1}^{n}\left(1-\eta_{j}\right)^{w_{j}} \leq 1-\prod_{j=1}^{n}\left(1-\eta^{+}\right)^{w_{j}}=\eta^{+} .
\end{gathered}
$$

That is,

$$
\begin{aligned}
& \frac{1}{\# h} \sum_{r_{j} \in h_{j}} \prod_{j=1}^{n}\left(r_{j}\right)^{w_{j}} \\
& \quad-\frac{1}{\# g} \sum_{\eta_{j} \in g_{j}}\left(1-\prod_{j=1}^{n}\left(1-\eta_{j}\right)^{w_{j}}\right) \geq r^{-}-\eta^{+},
\end{aligned}
$$


where $\# h$ and $\# g$ are the numbers of values in the membership degrees and nonmembership degrees of $\operatorname{DHFTLWG}\left(d_{1}, d_{2}, \ldots, d_{n}\right)$, respectively.

Since $s_{\theta^{-}}=\min _{1 \leq j \leq n} s_{\theta\left(d_{j}\right)}$, we have

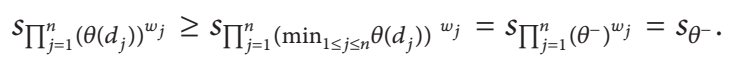

Similarly, we have

$$
s_{\prod_{j=1}^{n}\left(\pi\left(d_{j}\right)\right)^{w_{j}}} \geq s_{\pi^{-}}, \quad s_{\prod_{j=1}^{n}\left(\tau\left(d_{j}\right)\right)^{w_{j}}} \geq s_{\tau^{-}} .
$$
obtain

Therefore, according to Definition 9 and Theorem 11, we

$$
\left\langle\left[s_{\theta^{-}}, s_{\pi^{-}}, s_{\tau^{-}}\right],\left\{r^{-}\right\},\left\{\eta^{+}\right\}\right\rangle \leq \operatorname{DHFTLWG}\left(d_{1}, d_{2}, \ldots, d_{n}\right) .
$$

Similarly,

$$
\operatorname{DHFTLWG}\left(d_{1}, d_{2}, \ldots, d_{n}\right) \leq\left\langle\left[s_{\theta^{+}}, s_{\pi^{+}}, s_{\tau^{+}}\right],\left\{r^{+}\right\},\left\{\eta^{-}\right\}\right\rangle .
$$

Therefore,

$$
\begin{aligned}
\left\langle\left[s_{\theta^{-}}, s_{\pi^{-}}, s_{\tau^{-}}\right],\left\{r^{-}\right\},\left\{\eta^{+}\right\}\right\rangle & \leq \operatorname{DHFTLWG}\left(d_{1}, d_{2}, \ldots, d_{n}\right) \\
& \leq\left\langle\left[s_{\theta^{+}}, s_{\pi^{+}}, s_{\tau^{+}}\right],\left\{r^{+}\right\},\left\{\eta^{-}\right\}\right\rangle .
\end{aligned}
$$

However, the DHFTLWG operator is not idempotent, which can be illustrated by the following example.

Example 15. Let $d_{1}=\left\langle\left[s_{4}, s_{5}, s_{6}\right],\{0.3,0.5\},\{0.2,0.3\}\right\rangle=d$ and $d_{2}=\left\langle\left[s_{4}, s_{5}, s_{6}\right],\{0.3,0.5\},\{0.2,0.3\}\right\rangle=d$ be two DHFTLEs; $w=(0.6,0.4)^{T}$ is the weight vector of $d_{j}(j=1,2)$; then by the DHFTLWG operator, we have

$$
\begin{aligned}
& \operatorname{DHFTLWG}\left(d_{1}, d_{2}\right) \\
& =\left\langle\left[s_{4}, s_{5}, s_{6}\right],\{0.3,0.368,0.408,0.5\},\right. \\
& \quad\{0.2,0.242,0.262,0.3\}\rangle .
\end{aligned}
$$

It is clear that $\operatorname{DHFTLWG}\left(d_{1}, d_{2}\right) \neq d$, therefore, the idempotency is not hold.

Lemma 16 (see [37]). Let $x_{j}>0, \lambda_{j}>0, j=1,2, \ldots, n$, and $\sum_{j=1}^{n} \lambda_{j}=1$; then

$$
\prod_{j=1}^{n} x_{j}^{\lambda_{j}} \leq \sum_{j=1}^{n} \lambda_{j} x_{j}
$$

with equality if and only if $x_{1}=x_{2}=\cdots=x_{n}$.

Theorem 17. Let $d_{j}=\left\langle\left[s_{\theta\left(d_{j}\right)}, s_{\pi\left(d_{j}\right)}, s_{\tau\left(d_{j}\right)}\right], h_{j}, g_{j}\right\rangle(j=1$, $2, \ldots, n)$ be a collection of DHFTLEs; $w=\left(w_{1}, w_{2}, \ldots, w_{n}\right)^{T}$ is the weight vector of $d_{j}(j=1,2, \ldots, n)$, such that $w_{j} \in[0,1]$ and $\sum_{j=1}^{n} w_{j}=1$. One has

$$
\operatorname{DHFTLWG}\left(d_{1}, d_{2}, \ldots, d_{n}\right) \leq \operatorname{DHFTLWA}\left(d_{1}, d_{2}, \ldots, d_{n}\right) \text {, }
$$

where DHFTLWA $\left(d_{1}, d_{2}, \ldots, d_{n}\right)$ denotes the dual hesitant fuzzy triangular linguistic weighted average (DHFTLWA) operator of $d_{j}(j=1,2, \ldots, n)$ proposed by Ju and Yang [38].

Proof. According to Lemma 16, for any $d_{j}=\left\langle\left[s_{\theta\left(d_{j}\right)}, s_{\pi\left(d_{j}\right)}\right.\right.$, $\left.\left.s_{\tau\left(d_{j}\right)}\right], h_{j}, g_{j}\right\rangle(j=1,2, \ldots, n)$, we have

$$
\begin{gathered}
s_{\prod_{j=1}^{n}\left(\theta\left(d_{j}\right)\right)^{w_{j}}} \leq s_{\sum_{j=1}^{n} w_{j} \theta\left(d_{j}\right)}, \\
s_{\prod_{j=1}^{n}\left(\pi\left(d_{j}\right)\right)^{w_{j}}} \leq s_{\sum_{j=1}^{n} w_{j} \pi\left(d_{j}\right)}, \\
s_{\prod_{j=1}^{n}\left(\tau\left(d_{j}\right)\right)^{w_{j}}} \leq s_{\sum_{j=1}^{n} w_{j} \tau\left(d_{j}\right)}, \\
\prod_{j=1}^{n}\left(r_{j}\right)^{w_{j}} \leq \sum_{j=1}^{n} w_{j} r_{j} \\
=1-\sum_{j=1}^{n} w_{j}\left(1-r_{j}\right) \leq 1-\prod_{j=1}^{n}\left(1-r_{j}\right)^{w_{j}}, \\
1-\prod_{j=1}^{n}\left(1-\eta_{j}\right)^{w_{j}} \geq 1-\sum_{j=1}^{n} w_{j}\left(1-\eta_{j}\right) \\
=\sum_{j=1}^{n} w_{j} \eta_{j} \geq \prod_{j=1}^{n}\left(\eta_{j}\right)^{w_{j} ;}
\end{gathered}
$$

then, we have

$$
\begin{aligned}
\frac{1}{\# h_{1}} \sum_{r_{j} \in h_{j}} \prod_{j=1}^{n}\left(r_{j}\right)^{w_{j}}-\frac{1}{\# g_{1}} \sum_{\eta_{j} \in g_{j}}\left(1-\prod_{j=1}^{n}\left(1-\eta_{j}\right)^{w_{j}}\right) \\
\leq \frac{1}{\# h_{2}} \sum_{r_{j} \in h_{j}}\left(1-\prod_{j=1}^{n}\left(1-r_{j}\right)^{w_{j}}\right) \\
\quad-\frac{1}{\# g_{2}} \sum_{\eta_{j} \in g_{j}} \prod_{j=1}^{n}\left(\eta_{j}\right)^{w_{j}},
\end{aligned}
$$

where $\# h_{1}$ and $\# g_{1}$ are the numbers of values in the membership degrees and nonmembership degrees of $\operatorname{DHFTLWG}\left(d_{1}, d_{2}, \ldots, d_{n}\right)$, respectively; $\# h_{2}$ and $\# g_{2}$ are the numbers of values in the membership degrees and nonmembership degrees of DHFTLWA $\left(d_{1}, d_{2}, \ldots, d_{n}\right)$, respectively.

Therefore, based on Definition 9 and Theorem 11, we can obtain

$$
\begin{aligned}
& S\left(\operatorname{DHFTLWG}\left(d_{1}, d_{2}, \ldots, d_{n}\right)\right) \\
& \quad \leq S\left(\operatorname{DHFTLWA}\left(d_{1}, d_{2}, \ldots, d_{n}\right)\right) .
\end{aligned}
$$

which implies that $\operatorname{DHFTLWG}\left(d_{1}, d_{2}, \ldots, d_{n}\right) \leq$ $\operatorname{DHFTLWA}\left(d_{1}, d_{2}, \ldots, d_{n}\right)$.

Definition 18. Let $d_{j}=\left\langle\left[s_{\theta\left(d_{j}\right)}, s_{\pi\left(d_{j}\right)}, s_{\tau\left(d_{j}\right)}\right], h_{j}, g_{j}\right\rangle(j=$ $1,2, \ldots, n)$ be a collection of DHFTLEs, and DHFTLOWG : $\Omega^{n} \rightarrow \Omega$; then the dual hesitant fuzzy triangular linguistic 
ordered weighted geometric (DHFTLOWG) operator can be defined as

$$
\operatorname{DHFTLOWG}\left(d_{1}, d_{2}, \ldots, d_{n}\right)=\prod_{j=1}^{n}\left(d_{\sigma(j)}\right)^{\omega_{j}},
$$

in which $\Omega$ is a dual hesitant fuzzy triangular linguistic set and $\omega=\left(\omega_{1}, \omega_{2}, \ldots, \omega_{n}\right)^{T}$ is the aggregation-associated weight vector, such that $\omega_{j} \in[0,1]$ and $\sum_{j=1}^{n} \omega_{j}=1 . d_{\sigma(j)}=$ $\left\langle\left[s_{\theta_{\sigma(j)}}, s_{\pi_{\sigma(j)}}, s_{\tau_{\sigma(j)}}\right], h_{\sigma(j)}, g_{\sigma(j)}\right\rangle$ is the $j$ th largest element in $d_{j}$ $(j=1,2, \ldots, n)$.

Especially, if $\omega=(1 / n, 1 / n, \ldots, 1 / n)^{T}$, then the DHFTLOWG operator reduces to the dual hesitant fuzzy triangular linguistic geometric (DHFTLG) operator.

Theorem 19. Let $d_{j}=\left\langle\left[s_{\theta\left(d_{j}\right)}, s_{\pi\left(d_{j}\right)}, s_{\tau\left(d_{j}\right)}\right], h_{j}, g_{j}\right\rangle(j=$ $1,2, \ldots, n)$ be a collection of DHFTLEs; $\omega=\left(\omega_{1}, \omega_{2}, \ldots, \omega_{n}\right)^{T}$ is the aggregation-associated weight vector, such that $\omega_{j} \in$ $[0,1]$ and $\sum_{j=1}^{n} \omega_{j}=1$. Then their aggregated value by the DHFTLOWG operator is still a DHFTLE, and

$$
\begin{aligned}
& \operatorname{DHFTLOWG}\left(d_{1}, d_{2}, \ldots, d_{n}\right)
\end{aligned}
$$

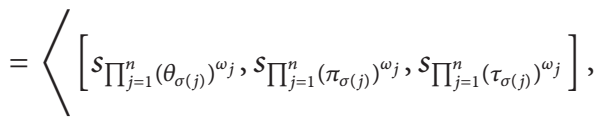

$$
\begin{aligned}
& \bigcup_{r_{\sigma(j)} \in h_{\sigma(j)}, \eta_{\sigma(j)} \in g_{\sigma(j)}}\left\{\left\{\prod_{j=1}^{n}\left(r_{\sigma(j)}\right)^{\omega_{j}}\right\},\right. \\
& \left.\left.\left\{1-\prod_{j=1}^{n}\left(1-\eta_{\sigma(j)}\right)^{\omega_{j}}\right\}\right\}\right\rangle \text {. }
\end{aligned}
$$

Theorem 20 (boundedness). Let $d_{j}=\left\langle\left[s_{\theta\left(d_{j}\right)}, s_{\pi\left(d_{j}\right)}\right.\right.$, $\left.\left.s_{\tau\left(d_{j}\right)}\right], h_{j}, g_{j}\right\rangle(j=1,2, \ldots, n)$ be a collection of DHFTLEs, for the DHFTLOWG operator, if $s_{\theta^{-}}=\min _{1 \leq j \leq n} s_{\theta\left(d_{j}\right)}, s_{\pi^{-}}=$ $\min _{1 \leq j \leq n} s_{\pi\left(d_{j}\right)}, s_{\tau^{-}}=\min _{1 \leq j \leq n} s_{\tau\left(d_{j}\right)}, s_{\theta^{+}}=\max _{1 \leq j \leq n} s_{\theta\left(d_{j}\right)}$, $s_{\pi^{+}}=\max _{1 \leq j \leq n} s_{\pi\left(d_{j}\right)}, s_{\tau^{+}}=\max _{1 \leq j \leq n} s_{\tau\left(d_{j}\right)}, r^{-}=\min _{1 \leq j \leq n}\left\{r_{j} \mid\right.$ $\left.r_{j} \in h_{j}\right\}, r^{+}=\max _{1 \leq j \leq n}\left\{r_{j} \mid r_{j} \in h_{j}\right\}, \eta^{-}=\min _{1 \leq j \leq n}\left\{\eta_{j} \mid \eta_{j} \in\right.$ $\left.g_{j}\right\}, \eta^{+}=\max _{1 \leq j \leq n}\left\{\eta_{j} \mid \eta_{j} \in g_{j}\right\}$, for all $j=1,2, \ldots, n$; then we can obtain

$$
\begin{aligned}
\left\langle\left[s_{\theta^{-}}, s_{\pi^{-}}, s_{\tau^{-}}\right],\left\{r^{-}\right\},\left\{\eta^{+}\right\}\right\rangle & \leq \operatorname{DHFTLOWG}\left(d_{1}, d_{2}, \ldots, d_{n}\right) \\
& \leq\left\langle\left[s_{\theta^{+}}, s_{\pi^{+}}, s_{\tau^{+}}\right],\left\{r^{+}\right\},\left\{\eta^{-}\right\}\right\rangle,
\end{aligned}
$$

which can be proven to be similar to Theorem 14.

Theorem 21 (commutativity). Let $d_{j}=\left\langle\left[s_{\theta\left(d_{j}\right)}, s_{\pi\left(d_{j}\right)}\right.\right.$, $\left.\left.s_{\tau\left(d_{j}\right)}\right], h_{j}, g_{j}\right\rangle(j=1,2, \ldots, n)$ be a collection of DHFTLEs, if $\left(d_{1}^{\prime}, d_{2}^{\prime}, \ldots, d_{n}^{\prime}\right)$ is any permutation of $\left(d_{1}, d_{2}, \ldots, d_{n}\right)$; then

$$
\begin{aligned}
& \operatorname{DHFTLOWG}\left(d_{1}^{\prime}, d_{2}^{\prime}, \ldots, d_{n}^{\prime}\right) \\
& \quad=\operatorname{DHFTLOWG}\left(d_{1}, d_{2}, \ldots, d_{n}\right) .
\end{aligned}
$$

Proof. Since $\left(d_{1}^{\prime}, d_{2}^{\prime}, \ldots, d_{n}^{\prime}\right)$ is a permutation of $\left(d_{1}, d_{2}\right.$, $\left.\ldots, d_{n}\right)$, we have $d_{\sigma(j)}^{\prime}=d_{\sigma(j)}$, for all $j=1,2, \ldots, n$. Then, based on Definition 18, we obtain

$$
\begin{aligned}
& \operatorname{DHFTLOWG}\left(d_{1}^{\prime}, d_{2}^{\prime}, \ldots, d_{n}^{\prime}\right) \\
& =\operatorname{DHFTLOWG}\left(d_{1}, d_{2}, \ldots, d_{n}\right),
\end{aligned}
$$

which completes the proof of Theorem 21.

Theorem 22. Let $d_{j}=\left\langle\left[s_{\theta\left(d_{j}\right)}, s_{\pi\left(d_{j}\right)}, s_{\tau\left(d_{j}\right)}\right], h_{j}, g_{j}\right\rangle(j=$ $1,2, \ldots, n)$ be a collection of DHFTLEs; $\omega=\left(\omega_{1}, \omega_{2}, \ldots, \omega_{n}\right)^{T}$ is the aggregation-associated weight vector, such that $\omega_{j} \in$ $[0,1]$ and $\sum_{j=1}^{n} \omega_{j}=1$. One has

$$
\begin{aligned}
& \operatorname{DHFTLOWG}\left(d_{1}, d_{2}, \ldots, d_{n}\right) \\
& \quad \leq \operatorname{DHFTLOWA}\left(d_{1}, d_{2}, \ldots, d_{n}\right),
\end{aligned}
$$

where DHFTLOWA $\left(d_{1}, d_{2}, \ldots, d_{n}\right)$ is the dual hesitant fuzzy triangular linguistic ordered weighted average (DHFTLOWA) operator of $d_{j}(j=1,2, \ldots, n)$ proposed by Ju and Yang [38]. This theorem can be proven to be similar to Theorem 17.

Definition 23. Let $d_{j}=\left\langle\left[s_{\theta\left(d_{j}\right)}, s_{\pi\left(d_{j}\right)}, s_{\tau\left(d_{j}\right)}\right], h_{j}, g_{j}\right\rangle(j=$ $1,2, \ldots, n)$ be a collection of DHFTLEs, and DHFTLHG : $\Omega^{n} \rightarrow \Omega$; then the dual hesitant fuzzy triangular linguistic hybrid geometric (DHFTLHG) operator can be defined as

$$
\operatorname{DHFTLHG}\left(d_{1}, d_{2}, \ldots, d_{n}\right)=\prod_{j=1}^{n}\left(\dot{d}_{\sigma(j)}\right)^{\omega_{j}},
$$

in which $\Omega$ is a dual hesitant fuzzy triangular linguistic set, $\omega=\left(\omega_{1}, \omega_{2}, \ldots, \omega_{n}\right)^{T}$ is the aggregation-associated weight vector, such that $\omega_{j} \in[0,1]$ and $\sum_{j=1}^{n} \omega_{j}=1 . \dot{d}_{\sigma(j)}$ is the $j$ th largest element in $\dot{d}_{j}\left(\dot{d}_{j}=d_{j}^{n w_{j}}, j=1,2, \ldots, n\right), w=$ $\left(w_{1}, w_{2}, \ldots, w_{n}\right)^{T}$ is the weight vector of $d_{j}(j=1,2, \ldots, n)$, such that $w_{j} \in[0,1]$ and $\sum_{j=1}^{n} w_{j}=1$, and $n$ is the balancing coefficient.

Especially, if $\omega=(1 / n, 1 / n, \ldots, 1 / n)^{T}$, then the DHFTLHG operator reduces to the DHFTLWG operator in (11). If $w=(1 / n, 1 / n, \ldots, 1 / n)^{T}$, then the DHFTLHG operator reduces to the DHFTLOWG operator in (28).

Theorem 24. Let $d_{j}=\left\langle\left[s_{\theta\left(d_{j}\right)}, s_{\pi\left(d_{j}\right)}, s_{\tau\left(d_{j}\right)}\right], h_{j}, g_{j}\right\rangle(j=$ $1,2, \ldots, n)$ be a collection of DHFTLEs and let $\omega=$ $\left(\omega_{1}, \omega_{2}, \ldots, \omega_{n}\right)^{T}$ be the aggregation-associated weight vector, 
such that $\omega_{j} \in[0,1]$ and $\sum_{j=1}^{n} \omega_{j}=1$. Then their aggregated value by the DHFTLHG operator is still a DHFTLE, and

$$
\begin{aligned}
& \operatorname{DHFTLHG}\left(d_{1}, d_{2}, \ldots, d_{n}\right) \\
& =\left\langle\left[ s_{\prod_{j=1}^{n}\left(\theta\left(\dot{d}_{\sigma(j)}\right)\right)^{\omega}}, s_{\left.\prod_{j=1}^{n}\left(\pi\left(\dot{d}_{\sigma(j)}\right)\right)^{\omega_{j}}, s_{\prod_{j=1}^{n}\left(\tau\left(\dot{d}_{\sigma(j)}\right)\right)^{\omega_{j}}}\right],}\right.\right. \\
& \bigcup_{\dot{r}_{\sigma(j)} \in \dot{h}_{\sigma(j)}, \dot{\eta}_{\sigma(j)} \in \dot{g}_{\sigma(j)}}\left\{\left\{\prod_{j=1}^{n}\left(\dot{r}_{\sigma(j)}\right)^{\omega_{j}}\right\},\right. \\
& \left.\left.\left\{1-\prod_{j=1}^{n}\left(1-\dot{\eta}_{\sigma(j)}\right)^{\omega_{j}}\right\}\right\}\right\rangle .
\end{aligned}
$$

Theorem 25 (boundedness). Let $d_{j}=\left\langle\left[s_{\theta\left(d_{j}\right)}, s_{\pi\left(d_{j}\right)}\right.\right.$, $\left.\left.s_{\tau\left(d_{j}\right)}\right], h_{j}, g_{j}\right\rangle(j=1,2, \ldots, n)$ be a collection of DHFTLEs, for the DHFTLHG operator; if $s_{\theta^{-}}=\min _{1 \leq j \leq n} s_{\theta\left(d_{j}\right)}, s_{\pi^{-}}=$ $\min _{1 \leq j \leq n} s_{\pi\left(d_{j}\right)}, s_{\tau^{-}}=\min _{1 \leq j \leq n} s_{\tau\left(d_{j}\right)}, s_{\theta^{+}}=\max _{1 \leq j \leq n} s_{\theta\left(d_{j}\right)}$, $s_{\pi^{+}}=\max _{1 \leq j \leq n} s_{\pi\left(d_{j}\right)}, s_{\tau^{+}}=\max _{1 \leq j \leq n} s_{\tau\left(d_{j}\right)}, r^{-}=\min _{1 \leq j \leq n}\left\{r_{j} \mid\right.$ $\left.r_{j} \in h_{j}\right\}, r^{+}=\max _{1 \leq j \leq n}\left\{r_{j} \mid r_{j} \in h_{j}\right\}, \eta^{-}=\min _{1 \leq j \leq n}\left\{\eta_{j} \mid \eta_{j} \epsilon\right.$ $\left.g_{j}\right\}, \eta^{+}=\max _{1 \leq j \leq n}\left\{\eta_{j} \mid \eta_{j} \in g_{j}\right\}$, for all $j=1,2, \ldots, n$, then one can obtain

$$
\begin{aligned}
\left\langle\left[s_{\theta^{-}}, s_{\pi^{-}}, s_{\tau^{-}}\right],\left\{r^{-}\right\},\left\{\eta^{+}\right\}\right\rangle & \leq \operatorname{DHFTLHG}\left(d_{1}, d_{2}, \ldots, d_{n}\right) \\
& \leq\left\langle\left[s_{\theta^{+}}, s_{\pi^{+}}, s_{\tau^{+}}\right],\left\{r^{+}\right\},\left\{\eta^{-}\right\}\right\rangle,
\end{aligned}
$$

which can be proven to be similar to Theorem 14 .

Theorem 26. Let $d_{j}=\left\langle\left[s_{\theta\left(d_{j}\right)}, s_{\pi\left(d_{j}\right)}, s_{\tau\left(d_{j}\right)}\right], h_{j}, g_{j}\right\rangle(j=$ $1,2, \ldots, n)$ be a collection of DHFTLEs and let $\omega=$ $\left(\omega_{1}, \omega_{2}, \ldots, \omega_{n}\right)^{T}$ be the aggregation-associated weight vector, such that $\omega_{j} \in[0,1]$ and $\sum_{j=1}^{n} \omega_{j}=1$. One has

$$
\operatorname{DHFTLHG}\left(d_{1}, d_{2}, \ldots, d_{n}\right) \leq \operatorname{DHFTLHA}\left(d_{1}, d_{2}, \ldots, d_{n}\right) \text {, }
$$

where DHFTLHA $\left(d_{1}, d_{2}, \ldots, d_{n}\right)$ is the dual hesitant fuzzy triangular linguistic hybrid average (DHFTLHA) operator of $d_{j}(j=1,2, \ldots, n)$ proposed by Ju and Yang [38]. This theorem can be proven similar to Theorem 17.

Definition 27. Let $d_{j}=\left\langle\left[s_{\theta\left(d_{j}\right)}, s_{\pi\left(d_{j}\right)}, s_{\tau\left(d_{j}\right)}\right], h_{j}, g_{j}\right\rangle(j=$ $1,2, \ldots, n)$ be a collection of DHFTLEs, and GDHFTLWG : $\Omega^{n} \rightarrow \Omega$; then the generalized dual hesitant fuzzy triangular linguistic weighted geometric (GDHFTLWG) operator can be defined as

$$
\operatorname{GDHFTLWG}_{\lambda}\left(d_{1}, d_{2}, \ldots, d_{n}\right)=\frac{1}{\lambda}\left(\prod_{j=1}^{n}\left(\lambda d_{j}\right)^{w_{j}}\right),
$$

in which $\Omega$ is a dual hesitant fuzzy triangular linguistic set and $w=\left(w_{1}, w_{2}, \ldots, w_{n}\right)^{T}$ is the weight vector of $d_{j}(j=$ $1,2, \ldots, n)$, such that $w_{j} \in[0,1]$ and $\sum_{j=1}^{n} w_{j}=1, \lambda>0$.
Especially, if $\lambda=1$, then the GDHFTLWG operator reduces to the DHFTLWG operator in (11).

Theorem 28. Let $d_{j}=\left\langle\left[s_{\theta\left(d_{j}\right)}, s_{\pi\left(d_{j}\right)}, s_{\tau\left(d_{j}\right)}\right], h_{j}, g_{j}\right\rangle(j=$ $1,2, \ldots, n)$ be a collection of DHFTLEs and let $w=$ $\left(w_{1}, w_{2}, \ldots, w_{n}\right)^{T}$ be the weight vector of $d_{j}(j=1,2, \ldots, n)$, such that $w_{j} \in[0,1]$ and $\sum_{j=1}^{n} w_{j}=1, \lambda>0$. Then their aggregated value by the GDHFTLWG operator is still a DHFTLE, and

$$
\begin{aligned}
& \operatorname{GDHFTLWG}_{\lambda}\left(d_{1}, d_{2}, \ldots, d_{n}\right)
\end{aligned}
$$

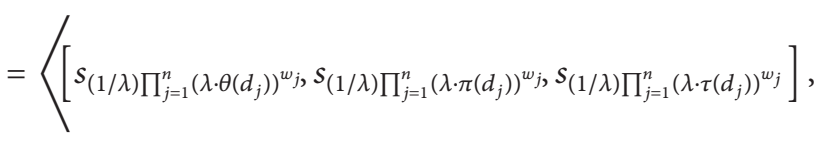

$$
\begin{aligned}
& \bigcup_{r_{j} \in h_{j}, \eta_{j} \in g_{j}}\left\{\left\{1-\left(1-\prod_{j=1}^{n}\left(1-\left(1-r_{j}\right)^{\lambda}\right)^{w_{j}}\right)^{1 / \lambda}\right\},\right. \\
& \left.\left.\left\{\left(1-\prod_{j=1}^{n}\left(1-\left(\eta_{j}\right)^{\lambda}\right)^{w_{j}}\right)^{1 / \lambda}\right\}\right\}\right\rangle \text {. }
\end{aligned}
$$

Theorem 29 (boundedness). Let $d_{j}=\left\langle\left[s_{\theta\left(d_{j}\right)}, s_{\pi\left(d_{j}\right)}\right.\right.$, $\left.\left.s_{\tau\left(d_{j}\right)}\right], h_{j}, g_{j}\right\rangle(j=1,2, \ldots, n)$ be a collection of DHFTLEs, for the GDHFTLWG operator; if $s_{\theta^{-}}=\min _{1 \leq j \leq n} s_{\theta\left(d_{j}\right)}, s_{\pi^{-}}=$ $\min _{1 \leq j \leq n} s_{\pi\left(d_{j}\right)}, s_{\tau^{-}}=\min _{1 \leq j \leq n} s_{\tau\left(d_{j}\right)}, s_{\theta^{+}}=\max _{1 \leq j \leq n} s_{\theta\left(d_{j}\right)}$, $s_{\pi^{+}}=\max _{1 \leq j \leq n} s_{\pi\left(d_{j}\right)}, s_{\tau^{+}}=\max _{1 \leq j \leq n} s_{\tau\left(d_{j}\right)}, r^{-}=\min _{1 \leq j \leq n}\left\{r_{j} \mid\right.$ $\left.r_{j} \in h_{j}\right\}, r^{+}=\max _{1 \leq j \leq n}\left\{r_{j} \mid r_{j} \in h_{j}\right\}, \eta^{-}=\min _{1 \leq j \leq n}\left\{\eta_{j} \mid \eta_{j} \epsilon\right.$ $\left.g_{j}\right\}, \eta^{+}=\max _{1 \leq j \leq n}\left\{\eta_{j} \mid \eta_{j} \in g_{j}\right\}$, for all $j=1,2, \ldots, n$; then one can obtain

$$
\begin{aligned}
\left\langle\left[s_{\theta^{-}}, s_{\pi^{-}}, s_{\tau^{-}}\right],\left\{r^{-}\right\},\left\{\eta^{+}\right\}\right\rangle & \leq \operatorname{GDHFTLWG} G_{\lambda}\left(d_{1}, d_{2}, \ldots, d_{n}\right) \\
& \leq\left\langle\left[s_{\theta^{+}}, s_{\pi^{+}}, s_{\tau^{+}}\right],\left\{r^{+}\right\},\left\{\eta^{-}\right\}\right\rangle
\end{aligned}
$$

which can be proven to be similar to Theorem 14.

Theorem 30. Let $d_{j}=\left\langle\left[s_{\theta\left(d_{j}\right)}, s_{\pi\left(d_{j}\right)}, s_{\tau\left(d_{j}\right)}\right], h_{j}, g_{j}\right\rangle(j=$ $1,2, \ldots, n)$ be a collection of DHFTLEs and let $w=$ $\left(w_{1}, w_{2}, \ldots, w_{n}\right)^{T}$ be the weight vector of $d_{j}(j=1,2, \ldots, n)$, such that $w_{j} \in[0,1]$ and $\sum_{j=1}^{n} w_{j}=1, \lambda>0$. One has

$$
\begin{aligned}
& \operatorname{GDHFTLWG}_{\lambda}\left(d_{1}, d_{2}, \ldots, d_{n}\right) \\
& \quad \leq \operatorname{GDHFTLWA}_{\lambda}\left(d_{1}, d_{2}, \ldots, d_{n}\right),
\end{aligned}
$$

where GDHFTLWA $\left(d_{1}, d_{2}, \ldots, d_{n}\right)$ is the generalized dual hesitant fuzzy triangular linguistic weighted average (GDHFTLWA) operator of $d_{j}(j=1,2, \ldots, n)$ proposed by $J u$ and Yang [38]. This theorem can be proven to be similar to Theorem 17.

Definition 31. Let $d_{j}=\left\langle\left[s_{\theta\left(d_{j}\right)}, s_{\pi\left(d_{j}\right)}, s_{\tau\left(d_{j}\right)}\right], h_{j}, g_{j}\right\rangle(j=$ $1,2, \ldots, n)$ be a collection of DHFTLEs, and GDHFTLOWG : 
$\Omega^{n} \rightarrow \Omega$; then the generalized dual hesitant fuzzy triangular linguistic ordered weighted geometric (GDHFTLOWG) operator can be defined as

$$
\operatorname{GDHFTLOWG}_{\lambda}\left(d_{1}, d_{2}, \ldots, d_{n}\right)=\frac{1}{\lambda}\left(\prod_{j=1}^{n}\left(\lambda d_{\sigma(j)}\right)^{\omega_{j}}\right) \text {, }
$$

in which $\Omega$ is a dual hesitant fuzzy triangular linguistic set and $\omega=\left(\omega_{1}, \omega_{2}, \ldots, \omega_{n}\right)^{T}$ is the aggregation-associated weight vector, such that $\omega_{j} \in[0,1]$ and $\sum_{j=1}^{n} \omega_{j}=1, \lambda>0 . d_{\sigma(j)}$ is the $j$ th largest element in $d_{j}(j=1,2, \ldots, n)$.

Especially, if $\lambda=1$, then the GDHFTLOWG operator reduces to the DHFTLOWG operator in (28).

Theorem 32. Let $d_{j}=\left\langle\left[s_{\theta\left(d_{j}\right)}, s_{\pi\left(d_{j}\right)}, s_{\tau\left(d_{j}\right)}\right], h_{j}, g_{j}\right\rangle(j=$ $1,2, \ldots, n)$ be a collection of DHFTLEs, and let $\omega=$ $\left(\omega_{1}, \omega_{2}, \ldots, \omega_{n}\right)^{T}$ be the aggregation-associated weight vector, such that $\omega_{j} \in[0,1]$ and $\sum_{j=1}^{n} \omega_{j}=1, \lambda>0$. Then their aggregated value by the GDHFTLOWG operator is still a DHFTLE, and

$$
\begin{aligned}
& \operatorname{GDHFTLOWG}_{\lambda}\left(d_{1}, d_{2}, \ldots, d_{n}\right)
\end{aligned}
$$

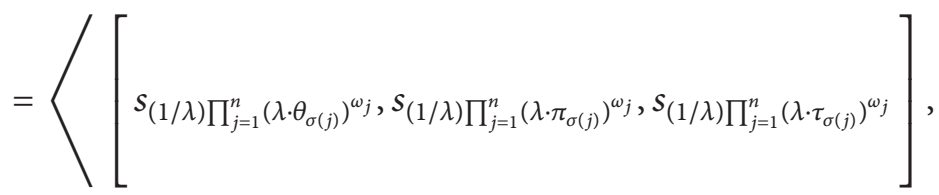

$$
\begin{aligned}
& \left.\bigcup_{r_{\sigma(j)} \in h_{\sigma(j)}, \eta_{\sigma(j)} \in g_{\sigma(j)}}\left\{\left\{1-\left(1-\prod_{j=1}^{n}\left(1-\left(1-r_{\sigma(j)}\right)^{\lambda}\right)^{\omega_{j}}\right)^{1 / \lambda}\right\},\left\{\left(1-\prod_{j=1}^{n}\left(1-\left(\eta_{\sigma(j)}\right)^{\lambda}\right)^{\omega_{j}}\right)^{1 / \lambda}\right\}\right\}\right\rangle .
\end{aligned}
$$

Theorem 33 (boundedness). Let $d_{j}=\left\langle\left[s_{\theta\left(d_{j}\right)}, s_{\pi\left(d_{j}\right)}\right.\right.$, $\left.\left.s_{\tau\left(d_{j}\right)}\right], h_{j}, g_{j}\right\rangle(j=1,2, \ldots, n)$ be a collection of DHFTLEs, for the GDHFTLOWG operator; if $s_{\theta^{-}}=\min _{1 \leq j \leq n} s_{\theta\left(d_{j}\right)}, s_{\pi^{-}}=$ $\min _{1 \leq j \leq n} s_{\pi\left(d_{j}\right)}, s_{\tau^{-}}=\min _{1 \leq j \leq n} s_{\tau\left(d_{j}\right)}, s_{\theta^{+}}=\max _{1 \leq j \leq n} s_{\theta\left(d_{j}\right)}$, $s_{\pi^{+}}=\max _{1 \leq j \leq n} s_{\pi\left(d_{j}\right)}, s_{\tau^{+}}=\max _{1 \leq j \leq n} s_{\tau\left(d_{j}\right)}, r^{-}=\min _{1 \leq j \leq n}\left\{r_{j} \mid\right.$ $\left.r_{j} \in h_{j}\right\}, r^{+}=\max _{1 \leq j \leq n}\left\{r_{j} \mid r_{j} \in h_{j}\right\}, \eta^{-}=\min _{1 \leq j \leq n}\left\{\eta_{j} \mid \eta_{j} \in\right.$ $\left.g_{j}\right\}, \eta^{+}=\max _{1 \leq j \leq n}\left\{\eta_{j} \mid \eta_{j} \in g_{j}\right\}$, for all $j=1,2, \ldots, n$, then one can obtain

$$
\begin{aligned}
& \left\langle\left[s_{\theta^{-}}, s_{\pi^{-}}, s_{\tau^{-}}\right],\left\{r^{-}\right\},\left\{\eta^{+}\right\}\right\rangle \\
& \quad \leq \operatorname{GDHFTLOWG} G_{\lambda}\left(d_{1}, d_{2}, \ldots, d_{n}\right) \\
& \quad \leq\left\langle\left[s_{\theta^{+}}, s_{\pi^{+}}, s_{\tau^{+}}\right],\left\{r^{+}\right\},\left\{\eta^{-}\right\}\right\rangle,
\end{aligned}
$$

which can be proven to be similar to Theorem 14.

Theorem 34 (commutativity). Let $d_{j}=\left\langle\left[s_{\theta\left(d_{j}\right)}, s_{\pi\left(d_{j}\right)}\right.\right.$, $\left.\left.s_{\tau\left(d_{j}\right)}\right], h_{j}, g_{j}\right\rangle(j=1,2, \ldots, n)$ be a collection of DHFTLEs; if $\left(d_{1}^{\prime}, d_{2}^{\prime}, \ldots, d_{n}^{\prime}\right)$ is any permutation of $\left(d_{1}, d_{2}, \ldots, d_{n}\right)$, then

$$
\begin{aligned}
& \operatorname{GDHFTLOWG}_{\lambda}\left(d_{1}^{\prime}, d_{2}^{\prime}, \ldots, d_{n}^{\prime}\right) \\
& =\operatorname{GDHFTLOWG}_{\lambda}\left(d_{1}, d_{2}, \ldots, d_{n}\right),
\end{aligned}
$$

which can be proven to be similar to Theorem 21.

Theorem 35. Let $d_{j}=\left\langle\left[s_{\theta\left(d_{j}\right)}, s_{\pi\left(d_{j}\right)}, s_{\tau\left(d_{j}\right)}\right], h_{j}, g_{j}\right\rangle(j=$ $1,2, \ldots, n)$ be a collection of DHFTLEs; $\omega=\left(\omega_{1}, \omega_{2}, \ldots, \omega_{n}\right)^{T}$ is the aggregation-associated weight vector, such that $\omega_{j} \in$ $[0,1]$ and $\sum_{j=1}^{n} \omega_{j}=1, \lambda>0$. One has

$$
\begin{aligned}
& \operatorname{GDHFTLOWG}_{\lambda}\left(d_{1}, d_{2}, \ldots, d_{n}\right) \\
& \leq \operatorname{GDHFTLOWA}_{\lambda}\left(d_{1}, d_{2}, \ldots, d_{n}\right)
\end{aligned}
$$

where GDHFTLOWA $\left(d_{1}, d_{2}, \ldots, d_{n}\right)$ is the generalized dual hesitant fuzzy triangular linguistic ordered weighted average (GDHFTLOWA) operator of $d_{j}(j=1,2, \ldots, n)$ proposed by $J u$ and Yang [38]. This theorem can be proven to be similar to Theorem 17.

\section{A Method for MADM Problems with Dual Hesitant Fuzzy Triangular Linguistic Information}

In this section, we will utilize the dual hesitant fuzzy triangular linguistic geometric aggregation operators to MADM for investment alternatives selection with dual hesitant fuzzy triangular linguistic information.

Let $A=\left\{A_{1}, A_{2}, \ldots, A_{m}\right\}$ be a finite set of $m$ alternatives, $C=\left\{C_{1}, C_{2}, \ldots, C_{n}\right\}$ the set of $n$ attributes, $S=\left\{s_{0}, s_{1}, \ldots, s_{l}\right\}$ a finite linguistic term set, and $w=\left(w_{1}, w_{2}, \ldots, w_{n}\right)^{T}$ the weight vector of attributes $C_{j}(j=1,2, \ldots, n)$, with $w_{j} \in$ $[0,1]$ and $\sum_{j=1}^{n} w_{j}=1$. Suppose that $D=\left(d_{i j}\right)_{m \times n}$ is a dual hesitant fuzzy triangular linguistic decision matrix, where $d_{i j}=\left\langle\left[s_{\theta\left(d_{i j}\right)}, s_{\pi\left(d_{i j}\right)}, s_{\tau\left(d_{i j}\right)}\right], h_{i j}, g_{i j}\right\rangle$ is in the form of DHFTLEs 
given for the alternative $A_{i}(i=1,2, \ldots, m)$ with respect to the attribute $C_{j}(j=1,2, \ldots, n)$, with $s_{\theta\left(d_{i j}\right)}, s_{\pi\left(d_{i j}\right)}, s_{\tau\left(d_{i j}\right)} \in S$, $h_{i j}=\bigcup_{r_{i j} \in h_{i j}}\left\{r_{i j}\right\}$ and $g_{i j}=\bigcup_{\eta_{i j} \in g_{i j}}\left\{\eta_{i j}\right\}$. Then, to determine the most desirable alternative(s), the DHFTLWG operator is utilized to develop a MADM method with dual hesitant fuzzy triangular linguistic information, which involves the following steps.
Step 1. Transform the dual hesitant fuzzy triangular linguistic decision matrix $D=\left(d_{i j}\right)_{m \times n}(i=1,2, \ldots, m, j=$ $1,2, \ldots, n)$ into normalized dual hesitant fuzzy triangular linguistic decision matrix $D^{\prime}=\left(d_{i j}^{\prime}\right)_{m \times n}$. The normalized method is given as follows:

$$
\begin{aligned}
d_{i j}^{\prime} & =\left\langle\left[s_{\theta\left(d_{i j}^{\prime}\right)}, s_{\pi\left(d_{i j}^{\prime}\right)}, s_{\tau\left(d_{i j}^{\prime}\right)}\right], h_{i j}^{\prime}, g_{i j}^{\prime}\right\rangle \\
& = \begin{cases}\left\langle\left[s_{\theta\left(d_{i j}\right)}, s_{\pi\left(d_{i j}\right)}, s_{\tau\left(d_{i j}\right)}\right], \bigcup_{r_{i j} \in h_{i j}}\left\{r_{i j}\right\}, \bigcup_{\eta_{i j} \in g_{i j}}\left\{\eta_{i j}\right\}\right\rangle, & j \in \Omega_{B} \\
\left\langle\left[s_{l-\theta\left(d_{i j}\right)}, s_{l-\pi\left(d_{i j}\right)}, s_{l-\tau\left(d_{i j}\right)}\right], \bigcup_{r_{i j} \in h_{i j}}\left\{1-r_{i j}\right\}, \bigcup_{\eta_{i j} \in G_{i j}}\left\{1-\eta_{i j}\right\}\right\rangle, & j \in \Omega_{C},\end{cases}
\end{aligned}
$$

where $\Omega_{B}$ and $\Omega_{C}$ are the sets of benefit attributes and cost attributes, respectively, and $l+1$ is the cardinality of linguistic term set $S$.

Step 2. Aggregate all assessment values $d_{i j}^{\prime}=\left\langle\left[s_{\theta\left(d_{i j}^{\prime}\right)}, s_{\pi\left(d_{i j}^{\prime}\right)}\right.\right.$, $\left.\left.s_{\tau\left(d_{i j}^{\prime}\right)}\right], h_{i j}^{\prime}, g_{i j}^{\prime}\right\rangle$ of the alternative $A_{i}(i=1,2, \ldots, m)$ on all attributes $C_{j}(j=1,2, \ldots, n)$ into the overall assessment values $d_{i}=\left\langle\left[s_{\theta\left(d_{i}\right)}, s_{\pi\left(d_{i}\right)}, s_{\tau\left(d_{i}\right)}\right], h_{i}, g_{i}\right\rangle$, based on the DHFTLWG operator in (48):

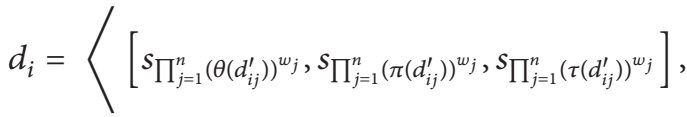

$$
\begin{aligned}
& \bigcup_{r_{i j}^{\prime} \in h_{i j}^{\prime}, \eta_{i j}^{\prime} \in g_{i j}^{\prime}}\left\{\left\{\prod_{j=1}^{n}\left(r_{i j}^{\prime}\right)^{w_{j}}\right\},\right. \\
& \left.\left.\left\{1-\prod_{j=1}^{n}\left(1-\eta_{i j}^{\prime}\right)^{w_{j}}\right\}\right\}\right\rangle,
\end{aligned}
$$

where $h_{i}=\bigcup_{r_{i} \in h_{i}}\left\{r_{i}\right\}$ and $g_{i}=\bigcup_{\eta_{i} \in g_{i}}\left\{\eta_{i}\right\}$.

Step 3. Calculate the score values $S\left(d_{i}\right)$ of the overall assessment values $d_{i}(i=1,2, \ldots, m)$ by (49):

$$
\begin{aligned}
S\left(d_{i}\right)= & \frac{\theta\left(d_{i}\right)+2 \pi\left(d_{i}\right)+\tau\left(d_{i}\right)}{4 l} \\
& \times\left(\frac{1}{\# h_{i}} \sum_{r_{i} \in h_{i}} r_{i}-\frac{1}{\# g_{i}} \sum_{\eta_{i} \in g_{i}} \eta_{i}\right),
\end{aligned}
$$

where $\# h_{i}$ and $\# g_{i}$ are the numbers of values in $h_{i}$ and $g_{i}$, respectively. If there is no difference between two score values $S\left(d_{i}\right)$ and $S\left(d_{t}\right)$, then we need to calculate the accuracy values $P\left(d_{i}\right)$ and $P\left(d_{t}\right)$ of the alternatives $A_{i}$ and $A_{t}(i, t=$ $1,2, \ldots, m)$, respectively, by $(50)$ :

$$
\begin{aligned}
P\left(d_{i}\right)= & \frac{\theta\left(d_{i}\right)+2 \pi\left(d_{i}\right)+\tau\left(d_{i}\right)}{4 l} \\
& \times\left(\frac{1}{\# h_{i}} \sum_{r_{i} \in h_{i}} r_{i}+\frac{1}{\# g_{i}} \sum_{\eta_{i} \in g_{i}} \eta_{i}\right) .
\end{aligned}
$$

Step 4. Rank all feasible alternatives $A_{i}(i=1,2, \ldots, m)$ according to Theorem 11 and select the most desirable alternative(s).

Step 5. End.

\section{Numerical Example}

In this section, a MADM problem adapted from Herrera and Herrera-Viedma [34] is used to illustrate the application of the MADM method proposed in Section 5 and to demonstrate its feasibility and effectiveness in a realistic scenario. An investment company wants to invest a sum of money in the best option. There is a panel with four possible alternatives: (1) $A_{1}$ is a car company; (2) $A_{2}$ is a food company; (3) $A_{3}$ is a computer company; (4) $A_{4}$ is an arms company. The investment company must make a decision according to the following three attributes: (1) $C_{1}$ is the market share analysis; (2) $C_{2}$ is the market growth analysis; (3) $C_{3}$ is the benefit analysis. The attribute weight vector is given as $w=$ $(0.35,0.25,0.40)^{T}$. The four possible alternatives $\left\{A_{1}, A_{2}, A_{3}\right.$, $A_{4}$ \} are evaluated by using the dual hesitant fuzzy triangular linguistic information under the above three attributes with the linguistic term set $S=\left\{s_{0}=\right.$ extremely low, $s_{1}=$ very low, $s_{2}=$ low, $s_{3}=$ medium, $s_{4}=$ high, $s_{5}=$ very high, $s_{6}$ $=$ extremely high $\}$, then the dual hesitant fuzzy triangular linguistic decision matrix $D=\left(d_{i j}\right)_{4 \times 3}$ is constructed as shown in Table 1. 
TABle 1: Dual hesitant fuzzy triangular linguistic decision matrix.

\begin{tabular}{cccc}
\hline & $C_{1}$ & $C_{2}$ & $C_{3}$ \\
\hline$A_{1}$ & $\left\langle\left[s_{2}, s_{3}, s_{4}\right],\{0.4,0.5,0.6\},\{0.3,0.4\}\right\rangle$ & $\left\langle\left[s_{3}, s_{4}, s_{5}\right],\{\{0.3,0.5\},\{0.2,0.3\}\}\right\rangle$ & $\left\langle\left[s_{3}, s_{4}, s_{5}\right],\{\{0.3,0.5,0.6\},\{0.1,0.2\}\}\right\rangle$ \\
$A_{2}$ & $\left\langle\left[s_{1}, s_{2}, s_{3}\right],\{\{0.4,0.5\},\{0.3,0.4\}\}\right\rangle$ & $\left\langle\left[s_{4}, s_{5}, s_{6}\right],\{\{0.4,0.5\},\{0.4,0.5\}\}\right\rangle$ & $\left\langle\left[s_{2}, s_{3}, s_{4}\right],\{\{0.2,0.5,0.6\},\{0.2,0.4\}\}\right\rangle$ \\
$A_{3}$ & $\left\langle\left[s_{3}, s_{4}, s_{5}\right],\{\{0.5,0.7\},\{0.2,0.3\}\}\right\rangle$ & $\left\langle\left[s_{2}, s_{3}, s_{4}\right],\{\{0.2,0.4,0.5\},\{0.3,0.4\}\}\right\rangle$ & $\left\langle\left[s_{2}, s_{3}, s_{4}\right],\{\{0.4,0.5,0.7\},\{0.2,0.3\}\}\right\rangle$ \\
$A_{4}$ & $\left\langle\left[s_{3}, s_{4}, s_{5}\right],\{\{0.4,0.6,0.8\},\{0.1,0.2\}\}\right\rangle$ & $\left\langle\left[s_{1}, s_{2}, s_{3}\right],\{\{0.5,0.6\},\{0.2,0.4\}\}\right\rangle$ & $\left\langle\left[s_{4}, s_{5}, s_{6}\right],\{\{0.6,0.7\},\{0.1,0.3\}\}\right\rangle$ \\
\hline
\end{tabular}

Step 1. Since all the three attributes are benefit attributes; therefore they are unnecessary to be normalized; that is, $D^{\prime}=$ $\left(d_{i j}^{\prime}\right)_{4 \times 3}=D=\left(d_{i j}\right)_{4 \times 3}$.

Step 2. Aggregate all assessment values $d_{i j}^{\prime}=\left\langle\left[s_{\theta\left(d_{i j}^{\prime}\right)}, s_{\pi\left(d_{i j}^{\prime}\right)}\right.\right.$, $\left.\left.s_{\tau\left(d_{i j}^{\prime}\right)}\right], h_{i j}^{\prime}, g_{i j}^{\prime}\right\rangle$ of the alternative $A_{i}(i=1,2,3,4)$ on all attributes $C_{j}(j=1,2,3)$ into the overall assessment values $d_{i}=\left\langle\left[s_{\theta\left(d_{i}\right)}, s_{\pi\left(d_{i}\right)}, s_{\tau\left(d_{i}\right)}\right], h_{i}, g_{i}\right\rangle$ by $(48)$ :

$$
\begin{gathered}
d_{1}=\left\langle\left[s_{2.7108}, s_{3.7224}, s_{4.7287}\right],\right. \\
\{0.3224,0.3855,0.4109,0.3955,0.4729,0.5040, \\
0.3409,0.4076,0.4345,0.4181,0.5000,0.5329, \\
\quad 0.3568,0.4266,0.4547,0.4376,0.5233,0.5578\}, \\
\{0.1937,0.2263,0.2356,0.2665,0.2242, \\
\quad 0.2555,0.2645,0.2942\}\rangle ; \\
d_{2}=\left\langle\left[s_{2.2191}, s_{3.3254}, s_{4.3779}\right],\right. \\
\{0.3138,0.4325,0.4610,0.3431,0.4729,0.5040, \\
\quad 0.3318,0.4573,0.4874,0.3628,0.5000,0.5329\}, \\
\{0.3104,0.3764,0.3589,0.4203, \\
\quad 0.3364,0.4000,0.3831,0.4422\}\rangle ; \\
d_{3}=\left\langle\left[s_{2.2134}, s_{3.2237}, s_{4.2295}\right],\right.
\end{gathered}
$$$$
\{0.3205,0.3466,0.3899,0.4229,0.4573,0.5145 \text {, }
$$$$
0.4624,0.5000,0.5625,0.3487,0.3770,0.4241 \text {, }
$$$$
0.4601,0.4974,0.5596,0.5030,0.5439,0.6119\} \text {, }
$$$$
\{0.2416,0.2762,0.2870,0.3195 \text {, }
$$$$
0.2665,0.3000,0.3104,0.3419\}\rangle ;
$$$$
d_{4}=\left\langle\left[s_{2.1380}, s_{3.2777}, s_{4.3445}\right]\right. \text {, }
$$$$
\{0.5040,0.5320,0.5422,0.5722,0.5578,0.5887 \text {, }
$$$$
0.6000,0.6333,0.5994,0.6326,0.6447,0.6805\} \text {, }
$$$$
\{0.1414,0.2137,0.2347,0.2992 \text {, }
$$$$
0.1663,0.2365,0.2570,0.3195\}\rangle \text {. }
$$

Step 3. Calculate the score values $S\left(d_{i}\right)$ of the overall assessment values $d_{i}(i=1,2,3,4)$. According to (49), the score values of alternatives $A_{i}(i=1,2,3,4)$ are obtained as follows:

$$
\begin{array}{ll}
S\left(d_{1}\right)=0.1196, & S\left(d_{2}\right)=0.0303 \\
S\left(d_{3}\right)=0.0904, & S\left(d_{4}\right)=0.1940
\end{array}
$$

Step 4. Rank all feasible alternatives $A_{i}(i=1,2,3,4)$ according to the descending order of corresponding score values $S\left(d_{i}\right)(i=1,2,3,4)$. The ranking of all investment alternatives $A_{i}(i=1,2,3,4)$ is obtained as follows:

$$
A_{4}>A_{1}>A_{3}>A_{2}
$$

where the symbol " $>$ " means "superior to." Therefore, $A_{4}$ is the most desirable investment alternative.

\section{Conclusions}

In this paper, with respect to the multiattribute decision making (MADM) problems in which the attribute values take the form of dual hesitant fuzzy triangular linguistic elements, a novel MADM method is proposed. Firstly, the concept, operational laws, score function, and accuracy function of dual hesitant fuzzy triangular linguistic elements (DHFTLEs) are defined. Then, a series of dual hesitant fuzzy triangular linguistic geometric aggregation operators is developed for aggregating the DHFTLEs, such as dual hesitant fuzzy triangular linguistic weighted geometric (DHFTLWG) operator, dual hesitant fuzzy triangular linguistic ordered weighted geometric (DHFTLOWG) operator, dual hesitant fuzzy triangular linguistic hybrid geometric (DHFTLHG) operator, generalized dual hesitant fuzzy triangular linguistic weighted geometric (GDHFTLWG) operator, and generalized dual hesitant fuzzy triangular linguistic ordered weighted geometric (GDHFTLOWG) operator. Furthermore, some desirable properties of these operators are investigated in detail. Based on the proposed operators, an approach to multiple attribute decision making with dual hesitant fuzzy triangular linguistic information is proposed. Finally, a numerical example for investment alternative selection is given to illustrate the application of the proposed method. In future research, we will focus on researching the applications of the MADM methods with dual hesitant fuzzy triangular linguistic information.

\section{Conflict of Interests}

The authors declare that there is no conflict of interests regarding the publication of this paper. 


\section{Acknowledgments}

This research is supported by Program for New Century Excellent Talents in University (NCET-13-0037), Natural Science Foundation of China (nos. 70972007 and 71271049), and Beijing Municipal Natural Science Foundation (nos. 9102015 and 9133020).

\section{References}

[1] T. Y. Chou, S. T. Chou, and G. H. Tzeng, "Evaluating IT/IS investments: a fuzzy multi-criteria decision model approach," European Journal of Operational Research, vol. 173, no. 3, pp. 1026-1046, 2006.

[2] M. Casanovas and J. M. Merigó, "Fuzzy aggregation operators in decision making with Dempster-Shafer belief structure," Expert Systems with Applications, vol. 39, no. 8, pp. 7138-7149, 2012.

[3] L. Dimova, P. Sevastianov, and D. Sevastianov, "MCDM in a fuzzy setting: investment projects assessment application," International Journal of Production Economics, vol. 100, no. 1, pp. 10-29, 2006.

[4] P. Hildebrandt and T. Knoke, "Investment decisions under uncertainty-A methodological review on forest science studies," Forest Policy and Economics, vol. 13, no. 1, pp. 1-15, 2011.

[5] L. A. Zadeh, "Fuzzy sets," Information and Computation, vol. 8, pp. 338-353, 1965.

[6] K. T. Atanassov, "Intuitionistic fuzzy sets," Fuzzy Sets and Systems, vol. 20, no. 1, pp. 87-96, 1986.

[7] K. T. Atanassov, Intuitionistic Fuzzy Sets: Theory and Applications, Physica, Heidelberg, Germany, 1999.

[8] R. Y. Chen, "A problem-solving approach to product design using decision tree induction based on intuitionistic fuzzy," European Journal of Operational Research, vol. 196, no. 1, pp. 266-272, 2009.

[9] D. F. Li, "Some measures of dissimilarity in intuitionistic fuzzy structures," Journal of Computer and System Sciences, vol. 68, no. 1, pp. 115-122, 2004.

[10] D. F. Li, "Multiattribute decision making models and methods using intuitionistic fuzzy sets," Journal of Computer and System Sciences, vol. 70, no. 1, pp. 73-85, 2005.

[11] Z. S. Xu, "Some similarity measures of intuitionistic fuzzy sets and their applications to multiple attribute decision making," Fuzzy Optimization and Decision Making, vol. 6, no. 2, pp. 109121, 2007.

[12] Z. S. Xu, "Intuitionistic fuzzy hierarchical clustering algorithms," Journal of Systems Engineering and Electronics, vol. 20, no. 1, pp. 90-97, 2009.

[13] M. M. Xia, Z. S. Xu, and B. Zhu, "Some issues on intuitionistic fuzzy aggregation operators based on Archimedean t-conorm and t-norm," Knowledge-Based Systems, vol. 31, pp. 78-88, 2012.

[14] L. A. Zadeh, "The concept of a linguistic variable and its application to approximate reasoning. I," vol. 8, pp. 199-249, 1975.

[15] F. Herrera and L. Martínez, "A 2-tuple fuzzy linguistic representation model for computing with words," IEEE Transactions on Fuzzy Systems, vol. 8, no. 6, pp. 746-752, 2000.

[16] J. Lin, J. B. Lan, and Y. H. Lin, "Multi-attribute group decisionmaking method based on the aggregation operators of interval 2-tuple linguistic information," Journal of Jilin Normal University, vol. 1, pp. 5-9, 2009.
[17] Z. S. Xu, "Uncertain linguistic aggregation operators based approach to multiple attribute group decision making under uncertain linguistic environment," Information Sciences, vol. 168, no. 1-4, pp. 171-184, 2004.

[18] Z. S. Xu, "An approach based on similarity measure to multiple attribute decision making with trapezoid fuzzy linguistic variables," in Proceedings of the 2nd International Confernce on Fuzzy Systems and Knowledge Discovery (FSKD '05), vol. 3613, pp. 110-117, August 2005.

[19] J. Q. Wang and J. J. Li, "The multi-criteria group decision making method based on multi-granularity intuitionistic two semantics," Science \& Technology Information, vol. 33, pp. 8-9, 2009.

[20] P. D. Liu and F. Jin, "Methods for aggregating intuitionistic uncertain linguistic variables and their application to group decision making," Information Sciences, vol. 205, pp. 58-71, 2012.

[21] P. D. Liu, "Some geometric aggregation operators based on interval intuitionistic uncertain linguistic variables and their application to group decision making," Applied Mathematical Modelling, vol. 37, no. 4, pp. 2430-2444, 2013.

[22] V. Torra and Y. Narukawa, "On hesitant fuzzy sets and decision," in Proceedings of the 18th IEEE International Conference on Fuzzy Systems, pp. 1378-1382, Jeju Island, Republic of Korea, August 2009.

[23] V. Torra, "Hesitant fuzzy sets," International Journal of Intelligent Systems, vol. 25, no. 6, pp. 529-539, 2010.

[24] N. Chen, Z. S. Xu, and M. M. Xia, "Interval-valued hesitant preference relations and their applications to group decision making," Knowledge-Based Systems, vol. 37, pp. 528-540, 2013.

[25] G. W. Wei, X. F. Zhao, and R. Lin, "Some hesitant intervalvalued fuzzy aggregation operators and their applications to multiple attribute decision making," Knowledge-Based Systems, vol. 46, pp. 43-53, 2013.

[26] R. M. Rodriguez, L. Martinez, and F. Herrera, "Hesitant fuzzy linguistic term sets for decision making," IEEE Transactions on Fuzzy Systems, vol. 20, no. 1, pp. 109-119, 2012.

[27] R. Lin, X. F. Zhao, and G. X. Wei, "Models for selecting an ERP system with hesitant fuzzy linguistic information," Journal of Intelligent \& Fuzzy Systems, 2013.

[28] B. Zhu, Z. Xu, and M. Xia, "Dual hesitant fuzzy sets," Journal of Applied Mathematics, vol. 2012, Article ID 879629, 13 pages, 2012.

[29] J. Ye, "Correlation coefficient of dual hesitant fuzzy sets and its application to multiple attribute decision making," Applied Mathematical Modelling, vol. 38, no. 2, pp. 659-666, 2014.

[30] Y. F. Chen, X. D. Peng, G. H. Guan, and H. D. Jiang, "Approaches to multiple attribute decision making based on the correlation coefficient with dual hesitant fuzzy information," Journal of Intelligent \& Fuzzy Systems, 2013.

[31] H. J. Wang, X. F. Zhao, and G. W. Wen, "Dual hesitant fuzzy aggregation operators in multiple attribute decision making," Journal of Intelligent \& Fuzzy Systems, 2013.

[32] Y. B. Ju, X. Y. Liu, and S. H. Yang, "Interval-valued dual hesitant fuzzy aggregation operators and their applications to multiple attribute decision making," Journal of Intelligent \& Fuzzy Systems, 2013.

[33] F. Herrera, E. Herrera-Viedma, and J. L. Verdegay, "A model of consensus in group decision making under linguistic assessments," Fuzzy Sets and Systems, vol. 78, no. 1, pp. 73-87, 1996. 
[34] F. Herrera and E. Herrera-Viedma, "Linguistic decision analysis: steps for solving decision problems under linguistic information," Fuzzy Sets and Systems, vol. 115, no. 1, pp. 67-82, 2000.

[35] Z. S. Xu, "Group decision making with triangular fuzzy linguistic variables," in Intelligent Data Engineering and Automated Learning, vol. 4881 of Lecture Notes in Computer Science, pp. 1726, 2007.

[36] M. M. Xia and Z. S. Xu, "Hesitant fuzzy information aggregation in decision making," International Journal of Approximate Reasoning, vol. 52, no. 3, pp. 395-407, 2011.

[37] Z. S. Xu, "On consistency of the weighted geometric mean complex judgement matrix in AHP," European Journal of Operational Research, vol. 126, no. 3, pp. 683-687, 2000.

[38] Y. B. Ju and S. H. Yang, "Some aggregation operators with dual hesitant fuzzy triangular linguistic elements and their application to multiple attribute decision making," Technical Report, 2013. 


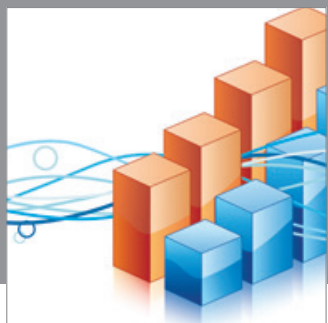

Advances in

Operations Research

mansans

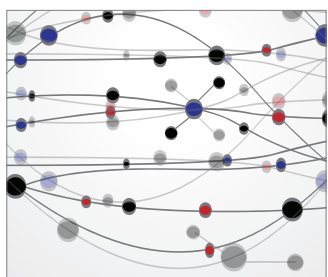

The Scientific World Journal
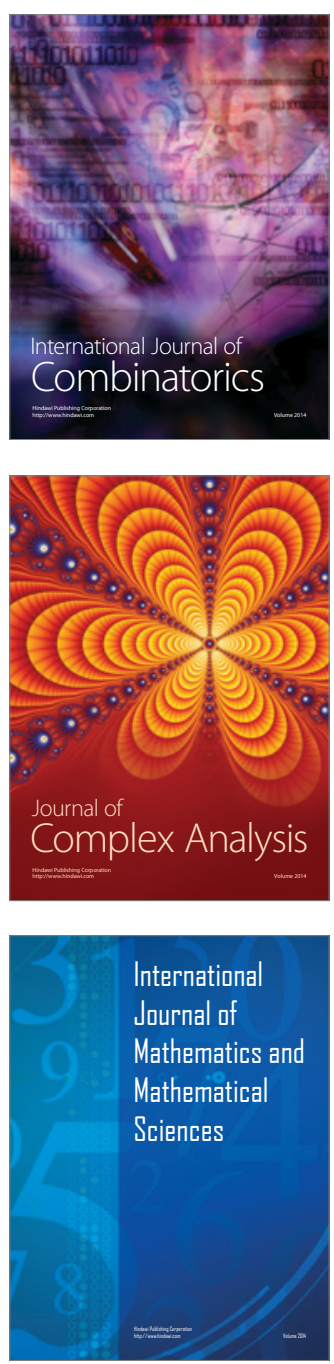
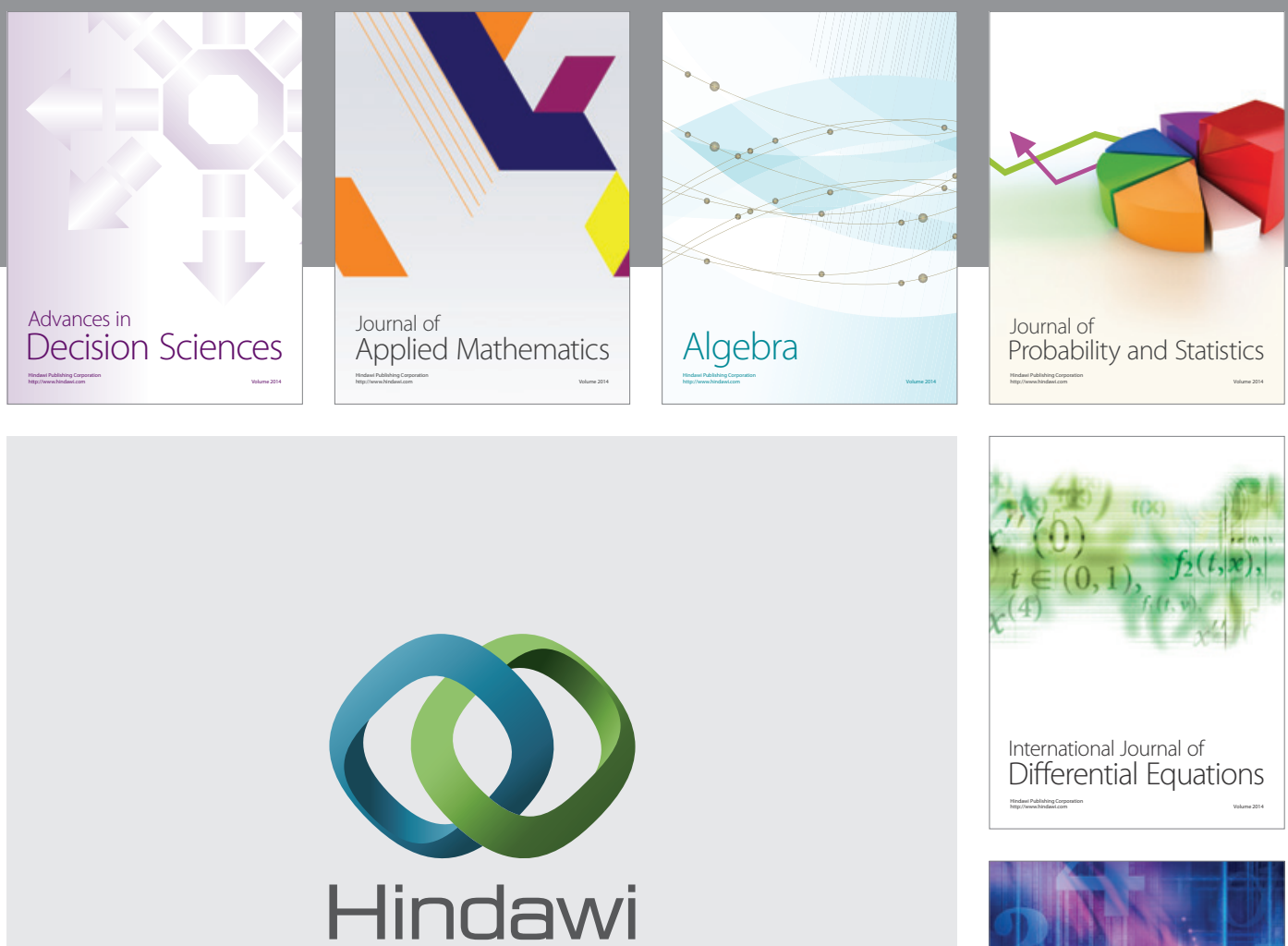

Submit your manuscripts at http://www.hindawi.com
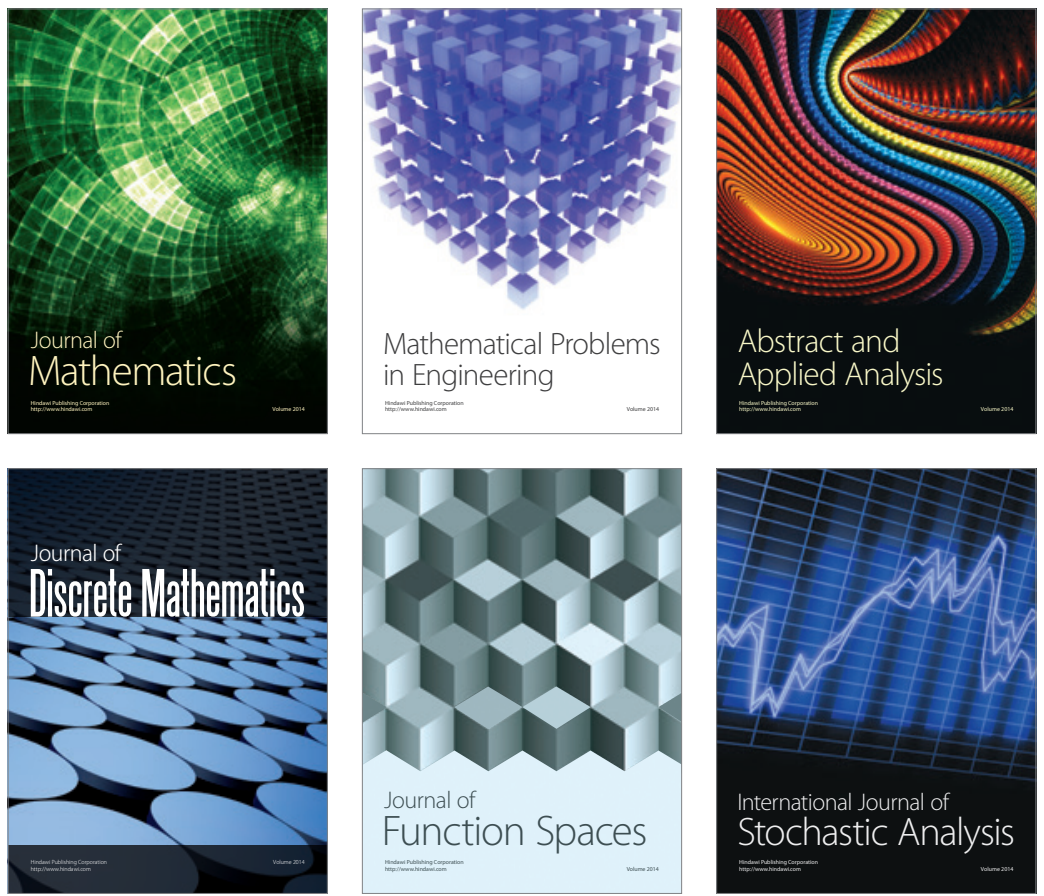

Journal of

Function Spaces

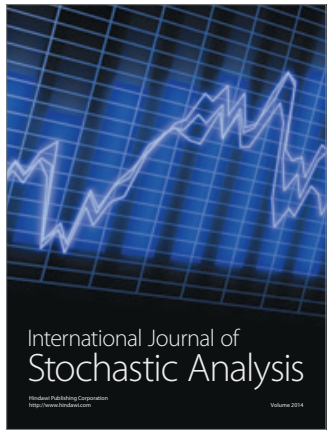

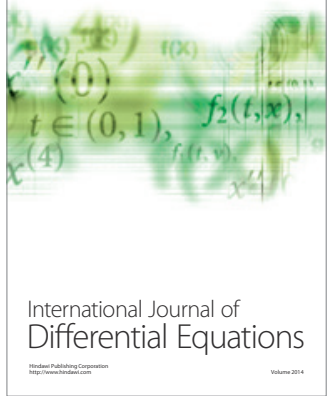
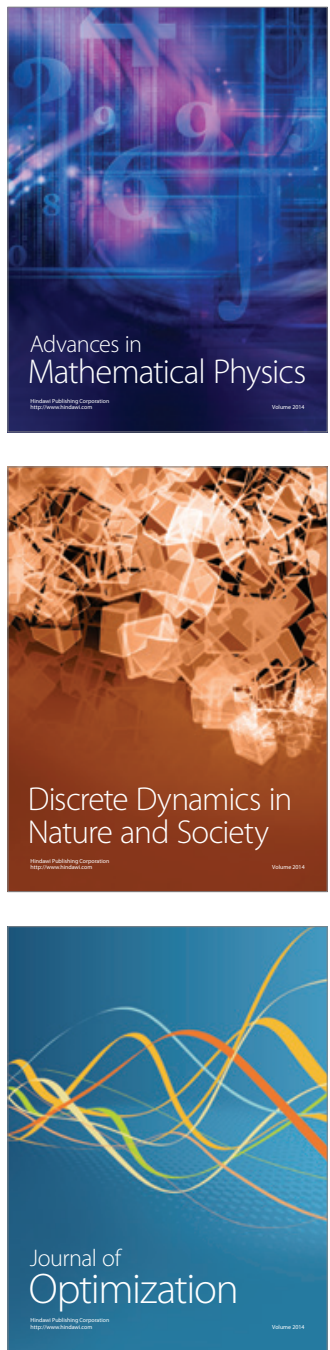